\title{
Fully discrete analysis of the heterogeneous multiscale method for elliptic problems with multiple scales
}

\author{
Assyr Abdulle ${ }^{1}$, Yun Bai ${ }^{1}$
}

\begin{abstract}
A fully discrete analysis of the finite element heterogeneous multiscale method (FEHMM) for elliptic problems with $N+1$ well separated scales is discussed. The FEHMM is a numerical homogenization method that relies on macroscopic scheme (macro FEM) for the approximation of the effective solution corresponding to the multiscale problem. The effective data are recovered from micro scale computation (micro FEM) on sampling domains located at appropriate quadrature points of the macroscopic mesh. At the macroscopic level, the numerical method can be seen as a FEM with numerical quadrature for a modified effective problem, hence, variational crimes are made when designing this method. Up to now, the method has been analyzed for two scales and the micro FEM was assumed to be conforming. For more than two scales, variational crimes are commited also at the intermediate (meso) scales and the effective data of the macroscopic scheme are obtained from a cascade of FEMs with numerical integration, which require a careful analysis. Numerical experiments for three scale problems illustrate the theoretical convergence rates.
\end{abstract}

Keywords: multiple scales, homogenization, heterogenenous multiscale method, numerical integration, variational crimes

AMS subject classification (2010): 65N30, 65M60, 35B27, 74Q05

\section{Introduction}

Numerical solutions of partial differential equations with multiples scales are basic problems for many applications, such as the conductivity of composite materials, stationary permeability in a porous media, etc. (see for example [20]). When the scale separation between the macroscopic scale of interest and the various microscopic scales where significant physical phenomena occur spans several order of magnitude, standard single scale numerical methods such as the finite element method (FEM) are usually too costly as the complexity scales with $\mathcal{O}\left(\varepsilon^{-d}\right)$, where $\varepsilon$ is the smallest scale in the problem and $d$ the spatial dimension of the physical problem. In recent years, a number of multiscale methods have been proposed to overcome the limitation of single scale methods (see $[18,15]$ and the references therein).

In this paper we consider numerical methods for a class of elliptic multiscale problems of the form

$$
\begin{aligned}
-\nabla \cdot\left(a^{\varepsilon}(x) \nabla u^{\varepsilon}(x)\right) & =f \text { in } \Omega, \\
u^{\varepsilon}(x) & =0 \text { on } \partial \Omega,
\end{aligned}
$$

${ }^{1}$ ANMC, Section de Mathématiques, École Polytechnique Fédérale de Lausanne, 1015 Lausanne, Switzerland, Assyr.Abdulle@epfl.ch,yun.bai@epfl.ch. 
in a domain $\Omega \in \mathbb{R}^{d}, d \leq 3$, where $a^{\varepsilon}=a\left(x, \frac{x}{\varepsilon_{1}}, \cdots, \frac{x}{\varepsilon_{N}}\right)$, and $\varepsilon_{1}, \ldots, \varepsilon_{N}$ are $N$ positive functions $\varepsilon_{i}(\varepsilon)$ that converge to 0 when $\varepsilon \rightarrow 0$ and that are well-separated in the sense that $\lim _{\varepsilon \rightarrow 0} \frac{\varepsilon_{i+1}(\varepsilon)}{\varepsilon_{i}(\varepsilon)}=0$ for $i=1, \ldots N-1$. The above tensor has thus one macroscopic scale and $N$ microscopic scales, i.e., it varies over $N+1$ scales (for simplicity of indexing the scales in our numerical scheme, we will refer to the macro scale as the zero-th scale). Here, we assume homogeneous Dirichlet boundary conditions for simplicity and take $f \in L^{2}(\Omega)$. As the problem (1) is often intractable numerically (we recall that the complexity of a standard FEM would be $\mathcal{O}\left(\varepsilon_{N}^{-d}\right)$ ), one usually looks for an effective equation, where the small scales have been averaged out.

Rigorous averaging theory for problems such as (1) has been derived in the framework of homogenization theory. We mention homogenization techniques using the multiple scale expansion [10, 22], whose convergence can be studied using the energy method due to Tartar [26]. Another approach is the two-scale convergence proposed by Nguentseng [24], developed and generalized by Allaire and Briane [8,9] for problems with more than two-scales.

Numerous numerical methods have been proposed recently for homogenization problems (see $[18,3,15,6]$ and the references therein). Most of the proposed methods for elliptic homogenization problems have however been proposed for two separated scales (a micro and a micro scale). A few methods have nevertheless been studied for elliptic problems with more than two scales. We mention an analysis of the multiscale finite element method by Efendiev et al. [19], and the numerical method based on high-dimensional FEM and sparse tensor-product approximation [21] based on the limit problem obtained from the reiterated homogenization proposed in [9].

In this paper we consider the finite element heterogeneous multiscale method introduced in [16]. This method has been analyzed in [17] (semi-discrete error analysis) and in [1,2] (fully discrete analysis) for two-scale problems (see also the reviews $[3,4]$ ). We propose a fully discrete analysis for $N+1$ scale problems. While the design of the FE-HMM for $N+1$ scale problems is straightforward from the method proposed in [16], the analysis is considerably more difficult than the analysis for two-scale problems $[17,1]$. Indeed, recall that this method is based on on macroscopic scheme (macro FEM) for the approximation of the effective solution corresponding to the considered multiscale problem. The effective data are then recovered by probing the fine scale and solving micro problems (micro FEM) around each macroscopic quadrature point. In turn, this method can be seen as a FEM with numerical quadrature for a modified effective problem. The data actually recovered by the micro FEMs are a perturbed version of the true effective data, because the computed data depends on the accuracy of the micro solver and on so-called modeling error (originating from the boundary conditions set for the micro problems and the size of the chosen sampling domains). For the analysis we have thus to deal with variational crimes (as we have a FEM with numerical quadrature) and modeling error (as the effective problem recovered differ from the true effective model). Yet for two-scale problems, the micro scale was assumed to be solved by conforming method in the analysis [1, 2], while for more than two scale problems, such assumption cannot be made as the problems at intermediate scales (called mesoscales in what follows) depend on effective coefficients computed around quadrature points of the meso FE meshes. Hence, we have a cascade of interdepending FEM with numerical quadrature and a cascade of variational crimes. In turn we need on one hand to generalize the analysis for FEM with numerical quadrature for single scale problem given by [12], on the other hand characterize the propagation of numerical discretization and modeling errors from micro to meso and macro scales. This precise characterization allows to set up the optimal meshes at each scale in order to obtain 
the desired convergence rate at the macro scale with minimal computational complexity. To avoid considerable notational difficulties, we will give the detailed analysis for three-scale problems. Its generalization to $N+1$ scale problems, that is conceptually not more difficult than the three-scale problem, will be sketched. We close this introduction by noting that a complexity analysis of the FE-HMM for $N+1$ scales shows that the method even though much cheaper than the fine scale problem (intractable in general) can be costly, due to the cascade of cell problems to be solved. However, as shown recently in [5], the use of reduced basis for the computations of the micro problems can considerably reduce the cost of repeated cell problems in the FE-HMM. The generalization of the results [5] to $N+1$ scale problems is a topic for further research. As for two-scale problems, it is likely that the analysis of the FE-HMM for $N+1$ scale problems will be an important ingredient for analyzing a reduced basis FE-HMM.

This paper is organized as follows. In Section 2, we briefly discuss the $N+1$ scale homogenization problem. The FE-HMM for $N+1$ scales is defined in Section 3. Existence, uniqueness and a priori error analysis are presented in Section 4. The modeling error of the FE-HMM for $N+1$ scales is discussed in Section 5. Numerical experiments in Section 6 and an appendix discussing results for the FEM with numerical quadrature conclude the paper.

\section{Model problem and homogenization}

We consider the model equation (1). Let $\Omega \subset \mathbb{R}^{d}$ be a bounded polyhedron subset in $\mathbb{R}^{d}$ and $f \in L^{2}(\Omega)$. Assume that $a^{\varepsilon}(x) \in L^{\infty}(\Omega)^{d \times d}$ is uniformly bounded and elliptic, i.e.,

$$
\lambda|\xi|^{2} \leq a^{\varepsilon} \xi \cdot \xi, \quad\left|a^{\varepsilon} \xi\right| \leq \Lambda|\xi|, \quad \forall \xi \in \mathbb{R}^{d}, \forall \varepsilon>0,
$$

for a.e. $x \in \Omega$. By Lax-Milgram theorem, there exists for all fixed $\varepsilon>0$ a unique solution $u^{\varepsilon}$ of (1) which is bounded in $H_{0}^{1}(\Omega)$ uniformly in $\varepsilon$. Hence, by standard compactness argument, one can show that there exists a subsequence of $\left\{u^{\varepsilon}\right\}$ that converges weakly in $H_{0}^{1}(\Omega)$. Invoking $H$ convergence [23] (or $G$ convergence [13] for the symmetric case) on can show that there exists a tensor $a^{0}(x) \in L^{\infty}(\Omega)^{d \times d}$ that is again elliptic and bounded and a subsequence of $\left\{u^{\varepsilon}\right\}$ that weakly converges in $H_{0}^{1}(\Omega)$ to $u_{0} \in H_{0}^{1}(\Omega)$ that is solution of the problem

$$
\begin{array}{rlll}
-\nabla \cdot\left(a^{0} \nabla u^{0}\right) & =f & \text { in } \Omega, \\
u^{0} & =0 & \text { on } \partial \Omega .
\end{array}
$$

However in the general case, the limit tensor $a^{0}$ is difficult to characterize and might not be unique. If one assumes that

$$
a^{\varepsilon}=a\left(x, \frac{x}{\varepsilon_{1}}, \cdots, \frac{x}{\varepsilon_{N}}\right)=a\left(x, y_{1}, \ldots, y_{N}\right) \in L^{\infty}\left(\Omega, C\left(\mathbb{R}^{d N}\right)\right)^{d \times d},
$$

that

$a$ is periodic with respect to $y_{s}$ with period $Y=[0,1]^{d}$ for each $s=1, \ldots, N$,

and that $\varepsilon_{1}, \varepsilon_{2}, \ldots, \varepsilon_{N}$ are $N$ positive functions $\varepsilon_{s}(\varepsilon)$ that converge to 0 when $\varepsilon \rightarrow 0$ and are well-separated

$$
\lim _{\varepsilon \rightarrow 0} \frac{\varepsilon_{s+1}(\varepsilon)}{\varepsilon_{s}(\varepsilon)}=0 \text { for } s=1, \ldots N-1,
$$


then as proved in [9], then the whole sequence $\left\{u^{\varepsilon}\right\}$ weakly converges in $H_{0}^{1}(\Omega)$ and the homogenized solution and homogenized tensor, $u^{0}, a^{0}$, respectively, are unique. Furthermore, $a^{0}(x)$ can be obtained by an inductive homogenization formula by computing a cascade of periodic micro functions and related homogenized tensors at the successive mesoscales (see $[9$, Corollary 2.12]). The FE-HMM for $N+1$ scales will be defined for a general tensor assuming $(2),(4)$ and (6). For a full characterization of the fine scale successive numerical errors we will in addition use (5). Finally assuming the periodicity of the tensor $a^{\varepsilon}(x)$ facilitates the analysis and will also be assumed here. We however note that this assumption could be removed at the cost of introducing dual problems to recover optimal convergence rates for the macro and micro scales (see [14] and [7, Lemma 4.6]).

\section{$3 \quad$ FE-HMM for $N+1$ scale problems}

In this section, we extend and analyse the FE-HMM discussed in $[17,1,3]$ (for two-scale problems) to $N+1$ scale problems.

The main idea is to apply a macro FEM to (3) and introduce mesoscopic FEMs on meso sampling domains to solve the meso cell problems recursively (recovering the missing data for the scale $s$ by cell problems at appropriate quadrature points at scale $s+1$ until the scale $N$ is reached where we use the given oscillatory data) in order to recover the unknown data in the macro solver (we emphasize that $a^{0}$ is unknown in general). This is illustrated in Fig. 1 for the three scale FE-HMM.

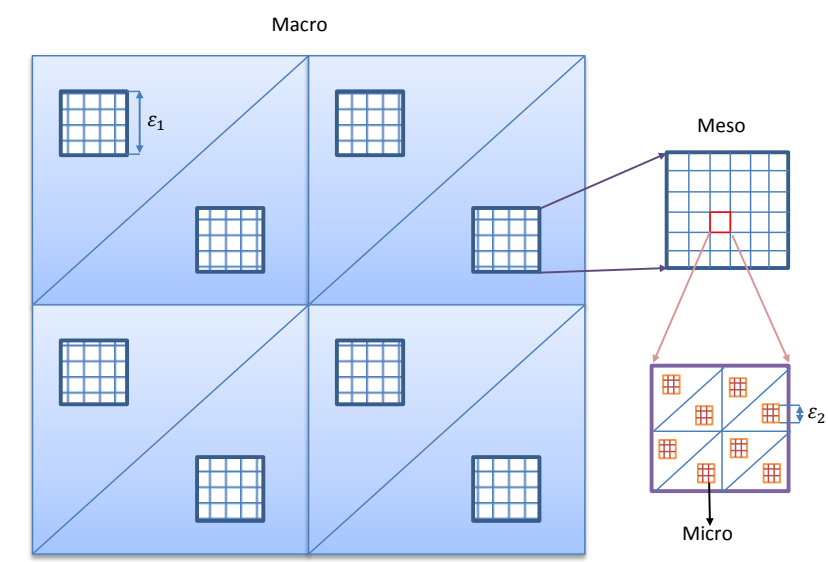

Figure 1: Illustration for the three scale FE-HMM

The FE-HMM is based on a macro finite element (FE) space

$$
S_{0}^{\ell}\left(\Omega, \mathcal{T}_{H}\right)=\left\{v^{H} \in H_{0}^{1}(\Omega) ;\left.v^{H}\right|_{K} \in \mathcal{R}^{\ell}(K), \forall K \in \mathcal{T}_{H}\right\},
$$

where $\mathcal{T}_{H}$ is a family of (macro) partition of $\Omega$ in simplicial or rectangular (parallelogram) elements $K$ of diameter $H_{K}$, and $\mathcal{R}^{\ell}(K)$ is the space $\mathcal{P}^{\ell}(K)$ of polynomials on $K$ of total 
degree at most $\ell$ if $K$ is a simplicial FE, or the space $\mathcal{Q}^{\ell}(K)$ of polynomials on $K$ of degree at most $\ell$ in each variable if $K$ is a rectangular (parallelogram) FE. For a given macro partition, we define as usual $H:=\max _{K \in \mathcal{T}_{H}} H_{K}$ and assume that the family of triangulation $\mathcal{T}_{H}$ is shape regular and satisfies the quasi-uniform assumption $\frac{H}{H_{K}} \leq C$ for all $K \in \mathcal{T}_{H}$ and all $\mathcal{T}_{H}$. We note that $H$ in our discretization is allowed to be much larger than $\varepsilon_{1}$.

We defining a quadrature formula $(\mathrm{QF})\left\{\hat{x}_{j}, \hat{\omega}_{j}\right\}_{j=1}^{J}$ on a reference element $\hat{K}$. We make the following assumptions on the quadrature formula, see [11]

$$
\begin{aligned}
& \text { (Q1) } \hat{\omega}_{j}>0, j=1, \ldots, J, \sum_{j=1}^{J} \hat{\omega}_{j}\left|\nabla \hat{p}\left(\hat{x}_{j}\right)\right|^{2} \geq \hat{\lambda}\|\nabla \hat{p}\|_{L^{2}(\hat{K})}^{2}, \forall \hat{p}(\hat{x}) \in \mathcal{R}^{r}(\hat{K}) \text {, with } \hat{\lambda}>0 ; \\
& \text { (Q2) } \int_{\hat{K}} \hat{p}(\hat{x}) d \hat{x}=\sum_{j \in J} \hat{\omega}_{j} \hat{p}\left(\hat{x}_{j}\right), \forall \hat{p}(\hat{x}) \in \mathcal{R}^{\sigma}(\hat{K}) .
\end{aligned}
$$

Each choice of $(J, r, \sigma)$ gives us a different $\mathrm{QF}$ which we will specify later when defining the FEMs for the meso and micro cell problems.

For a given $J_{1} \in \mathbb{N}$ and a given $\mathrm{QF}\left\{\hat{x}_{j}, \hat{\omega}_{j}\right\}_{j=1}^{J}\left(J=J_{1}\right)$, define the quadrature nodes on each macro element $K \in \mathcal{T}_{H}$ by the affine map $x_{K, j_{1}}=F_{K}\left(\hat{x}_{j_{1}}\right), j_{1}=1, \cdots, J_{1}$ and corresponding quadrature weights $\omega_{K, j_{1}}=|K| \hat{\omega}_{j_{1}}$.

We define the first meso scale sampling domains $\delta_{K, j_{1}}$ around each $x_{K, j_{1}}$ by

$$
\delta_{K, j_{1}}=x_{K, j_{1}}+\left(-\delta_{1} / 2, \delta_{1} / 2\right)^{d}, \text { with } \delta_{1} \geq \varepsilon_{1} .
$$

We then consider a partition $\mathcal{T}_{K, j_{1}}$ of $\delta_{K, j_{1}}$ with elements $T_{K, j_{1}}$ of size $h_{1}$. Likewise, we define recursively a sequence of sampling domains

$$
\delta_{K, j_{1}, \cdots, j_{s}}=x_{K, j_{1}, \cdots, j_{s}}+\left(-\delta_{s} / 2, \delta_{s} / 2\right)^{d}, \text { with } \delta_{s} \geq \varepsilon_{s}, s=1, \cdots, N,
$$

where $x_{K, j_{1}, \cdots, j_{s}}$ are the quadrature nodes on the element $T_{K, j_{1}, \cdots, j_{s-1}} \in \mathcal{T}_{K, j_{1}, \cdots, j_{s-1}}$ and $\mathcal{T}_{K, j_{1}, \cdots, j_{s-1}}$ is a partition of $\delta_{K, j_{1}, \cdots, j_{s-1}}$. Here the quadrature nodes are defined through an affine map

$$
x_{K, j_{1}, \cdots, j_{s}}=F_{K, j_{1}, \cdots, j_{s}}\left(\hat{x}_{j_{s}}\right), j_{s}=1, \cdots, J_{s}
$$

from the reference quadrature nodes $\left\{\hat{x}_{j}\right\}_{j=1}^{J}$ on $\hat{K}\left(J=J_{s}\right)$.

Now we define on scale $s$ an FE space $S^{q_{s}}\left(\delta_{K, j_{1}, \cdots, j_{s}}, \mathcal{T}_{K, j_{1}, \cdots, j_{s}}\right) \subset W\left(\delta_{K, j_{1}, \cdots, j_{s}}\right)$ with simplicial or rectangular FEs and piecewise polynomials of degree $q_{s}$, where $s=1, \cdots, N$. The space $W\left(\delta_{K, j_{1}, \cdots, j_{s}}\right)$ is the Sobolev space

$$
W\left(\delta_{K, j_{1}, \cdots, j_{s}}\right)=W_{p e r}^{1}\left(\delta_{K, j_{1}, \cdots, j_{s}}\right)=\left\{z \in H_{p e r}^{1}\left(\delta_{K, j_{1}, \cdots, j_{s}}\right) ; \int_{\delta_{K, j_{1}, \cdots, j_{s}}} z d x=0\right\}
$$

for a periodic coupling, where $H_{\text {per }}^{1}\left(\delta_{K, j_{1}, \cdots, j_{s}}\right):=\left\{g \in H^{1}\left(\delta_{K, j_{1}, \cdots, j_{s}}\right) \mid g\right.$ periodic in $\left.\delta_{K, j_{1}, \cdots, j_{s}}\right\}$, or

$$
W\left(\delta_{K, j_{1}, \cdots, j_{s}}\right)=H_{0}^{1}\left(\delta_{K, j_{1}, \cdots, j_{s}}\right)
$$

for a coupling with Dirichlet boundary conditions.

We next introduce meso and micro problems to recover an approximation of the unknown data $a^{0}(x)$ at suitable quadrature macro nodes.

Meso and micro problems. Assuming $a_{K, j_{1}, \cdots, j_{s}}^{s}\left(x_{K, j_{1}, \cdots, j_{s+1}}\right)$ is given, associated to each sampling domain $\delta_{K, j_{1}, \cdots, j_{s}}, s=1, \cdots, N-1,1 \leq j_{m} \leq J_{m}, m \leq s$, we define an effective 
numerical tensor $a_{K, j_{1}, \cdots, j_{s-1}}^{s-1}\left(x_{K, j_{1}, \cdots, j_{s}}\right)$ on the quadrature nodes $x_{K, j_{1}, \cdots, j_{s}}$ by

$$
\begin{aligned}
& a_{K, j_{1}, \cdots, j_{s-1}}^{s-1}\left(x_{K, j_{1}, \cdots, j_{s}}\right)=\frac{1}{\left|\delta_{K, j_{1}, \cdots, j_{s}}\right|} \sum_{T_{K, j_{1}, \cdots, j_{s}} \in \mathcal{T}_{K, j_{1}, \cdots, j_{s}}} \sum_{j_{s+1}=1}^{J_{s+1}} \omega_{K, j_{1}, \cdots, j_{s+1}} . \\
& a_{K, j_{1}, \cdots, j_{s}}^{s}\left(x_{K, j_{1}, \cdots, j_{s+1}}\right)\left(I+J_{\chi_{K, j_{1}, \cdots, j_{s}}^{h_{s}}\left(x_{K, j_{1}, \cdots, j_{s+1}}\right)}^{T}\right) \text {, }
\end{aligned}
$$

where $J_{\chi_{K, j_{1}, \cdots, j_{s}}^{h_{s}}(x)}$ is a $d \times d$ Jacobian matrix with entries $\left(J_{\chi_{K, j_{1}, \cdots, j_{s}}^{h_{s}}(x)}\right)_{i k}=\partial_{k}\left(\chi_{K, j_{1}, \cdots, j_{s}}^{i, h_{s}}(x)\right)$ $\left(\partial_{k}:=\partial / \partial x_{k}\right)$ and $\left|\delta_{K, j_{1}, \cdots, j_{s}}\right|$ denotes the measure of the sampling domain $\delta_{K, j_{1}, \cdots, j_{s}}$. Here we denote by $\chi_{K, j_{1}, \cdots, j_{s}}^{i, h_{s}}(x) \in S^{q_{s}}\left(\delta_{K, j_{1}, \cdots, j_{s}}, \mathcal{T}_{K, j_{1}, \cdots, j_{s}}\right)$ the solution of the following cell problem

$$
\begin{gathered}
\text { (PS) } \sum_{T_{K, j_{1}, \cdots, j_{s}} \in \mathcal{T}_{K, j_{1}, \cdots, j_{s}}} \sum_{j_{s+1}=1}^{J_{s+1}} \omega_{K, j_{1}, \cdots, j_{s+1}} a_{K, j_{1}, \cdots, j_{s}}^{s}\left(x_{K, j_{1}, \cdots, j_{s+1}}\right)\left(\nabla \chi_{K, j_{1}, \cdots, j_{s}}^{i, h_{s}}\left(x_{K, j_{1}, \cdots, j_{s+1}}\right)\right. \\
\left.+\mathbf{e}_{i}\right) \cdot \nabla z^{h_{s}}=0 \quad \forall z^{h_{s}} \in S^{q_{s}}\left(\delta_{K, j_{1}, \cdots, j_{s}}, \mathcal{T}_{K, j_{1}, \cdots, j_{s}}\right),
\end{gathered}
$$

where $\mathbf{e}_{i}, i=1, \cdots, d$ denote the canonical basis of $\mathbb{R}^{d}$. We note that $(\mathbf{P S})$ is defined recursively as the data $a_{K, j_{1}, \cdots, j_{s}}^{s}\left(x_{K, j_{1}, \cdots, j_{s+1}}\right)$ depends on $a_{K, j_{1}, \cdots, j_{s-1}}^{s-1}\left(x_{K, j_{1}, \cdots, j_{s}}\right)$, see $(9)^{1}$. The last level of the recursion is obtained by solving the micro problems on scale $N$, where we use the available microscopic data from (1).

The cell solution $\chi_{K, j_{1}, \cdots, j_{N}}^{i, h_{N}} \in S^{q_{N}}\left(\delta_{K, j_{1}, \cdots, j_{N}}, \mathcal{T}_{h_{N}}\right)$ on scale $N$ satisfies

$(\mathbf{P N}) \quad \int_{\delta_{K, j_{1}, \cdots, j_{N}}} a^{\varepsilon}(x)\left(\nabla \chi_{K, j_{1}, \cdots, j_{N}}^{i, h_{N}}+\mathbf{e}_{i}\right) \cdot \nabla z^{h_{N}} d x=0 \quad \forall z^{h_{N}} \in S^{q_{N}}\left(\delta_{K, j_{1}, \cdots, j_{N}}, \mathcal{T}_{h_{N}}\right)$.

The tensor $a_{K, j_{1}, \cdots, j_{N-1}}^{N-1}\left(x_{K, j_{1}, \cdots, j_{N}}\right)$ can be estimated as

$$
a_{K, j_{1}, \cdots, j_{N-1}}^{N-1}\left(x_{K, j_{1}, \cdots, j_{N}}\right)=\frac{1}{\left|\delta_{K, j_{1}, \cdots, j_{N}}\right|} \int_{\delta_{K, j_{1}, \cdots, j_{N}}} a^{\varepsilon}(x)\left(I+J_{\chi_{K, j_{1}, \cdots, j_{N}}(x)}^{T}\right) d x
$$

where $\left(J_{\chi_{K, j_{1}, \cdots, j_{N}}^{h_{N}}(x)}\right)_{i k}=\partial_{k}\left(\chi_{K, j_{1}, \cdots, j_{N}}^{i, h_{N}}(x)\right)$. We observe that the symmetry of $a^{\varepsilon}$ implies the symmetry of $a_{K, j_{1}, \cdots, j_{N-1}}^{N-1}\left(x_{K, j_{1}, \cdots, j_{N}}\right)$ and iteratively the symmetry of $a_{K, j_{1}, \cdots, j_{s}}^{s}\left(x_{K, j_{1}, \cdots, j_{s+1}}\right)$ $(s=0, \cdots, N-1)$.

Macro problem. The macro problem for the FE-HMM is defined as follows: find $u^{H} \in$ $S_{0}^{\ell}\left(\Omega, \mathcal{T}_{H}\right)$ such that

$$
B_{H}\left(u^{H}, v^{H}\right)=\int_{\Omega} f v^{H} d x \quad \forall v^{H} \in S_{0}^{\ell}\left(\Omega, \mathcal{T}_{H}\right),
$$

where

$$
B_{H}\left(u^{H}, v^{H}\right)=\sum_{K \in \mathcal{T}_{H}} \sum_{j_{1}=1}^{J_{1}} \omega_{K, j_{1}} a_{K}^{0}\left(x_{K, j_{1}}\right) \nabla u^{H}\left(x_{K, j_{1}}\right) \cdot \nabla v^{H}\left(x_{K, j_{1}}\right),
$$

\footnotetext{
${ }^{1}$ It will be proved in Section 4.3 that each of the problems (PS) has a unique solution.
} 
and $a_{K}^{0}\left(x_{K, j_{1}}\right)$ is the macro numerical homogenized tensor given by

$$
a_{K}^{0}\left(x_{K, j_{1}}\right):=\frac{1}{\left|\delta_{K, j_{1}}\right|} \sum_{T_{K, j_{1}} \in \mathcal{T}_{K, j_{1}}} \sum_{j_{2}=1}^{J_{2}} \omega_{K, j_{1}, j_{2}} a_{K, j_{1}}^{1}\left(x_{K, j_{1}, j_{2}}\right)\left(I+J_{\chi_{K, j_{1}}^{h_{1}}\left(x_{K, j_{1}, j_{2}}\right)}\right) .
$$

Here $\left.\left(J_{\chi_{K, j_{1}}^{h_{1}}(x)}\right)_{i k}=\partial_{k}\left(\chi_{K, j_{1}}^{i, h_{1}}(x)\right)\right)$ and $\chi_{K, j_{1}}^{i, h_{1}}$ is the solution of cell problem $(\mathbf{P S})(s=$ $1)$. We emphasize that the symmetry of $a_{K}^{0}\left(x_{K, j_{1}}\right)$ can be deduced from the symmetry of $a_{K, j_{1}}^{1}\left(x_{K, j_{1}, j_{2}}\right)$.

\section{A priori error analysis for the $N+1$ scale FE-HMM}

Our aim is to obtain the a priori errors $\left\|u^{H}-u^{0}\right\|_{L^{2}(\Omega)}$ and $\left\|u^{H}-u^{0}\right\|_{H^{1}(\Omega)}$ for the $N+1$ scale FE-HMM $(N \geq 2)$. Let us first show that the $N+1$ scale FE-HMM is well-defined.

\subsection{Existence and uniqueness of the FE-HMM solution.}

With the assumption (2), we have the following lemmas.

Lemma 4.1. Assume that the multiscale tensor $a^{\varepsilon}$ is symmetric, uniformly elliptic and bounded, i.e. (2) holds, then for any microscopic solution $\chi_{K, j_{1}, \cdots, j_{N}}^{i, h_{N}}$ of problem (PN), we have

$$
\sqrt{\left|\delta_{K, j_{1}, \cdots, j_{N}}\right|} \leq\left\|\mathbf{e}_{i}+\nabla \chi_{K, j_{1}, \cdots, j_{N}}^{i, h_{N}}\right\|_{L^{2}\left(\delta_{K, j_{1}, \cdots, j_{N}}\right)} \leq C \sqrt{\left|\delta_{K, j_{1}, \cdots, j_{N}}\right|} .
$$

Furthermore, we have

$$
a_{K, j_{1}, \cdots, j_{N-1}}^{N-1}\left(x_{K, j_{1}, \cdots, j_{N}}\right) \xi \cdot \xi \geq \lambda|\xi|^{2},\left|a_{K, j_{1}, \cdots, j_{N-1}}^{N-1}\left(x_{K, j_{1}, \cdots, j_{N}}\right) \xi\right| \leq \tilde{C}|\xi|, \quad \forall \xi \in \mathbb{R}^{d} .
$$

where constants $C, \tilde{C}>1$ depend on $\lambda, \Lambda$ and $d$.

Proof. We first show the upper bound of $\left\|e_{i}+\nabla \chi_{K, j_{1}, \cdots, j_{N}}^{i, h_{N}}(x)\right\|_{L^{2}\left(\delta_{K, j_{1}, \cdots, j_{N}}\right)}$. Using the symmetry of $a^{\varepsilon}(x)$ and $(\mathbf{P N})$, we can equivalently write (10) as follows

$$
\begin{aligned}
& a_{K, j_{1}, \cdots, j_{N-1}}^{N-1}\left(x_{K, j_{1}, \cdots, j_{N}}\right) \mathbf{e}_{i} \cdot \mathbf{e}_{k} \\
& =\frac{1}{\left|\delta_{K, j_{1}, \cdots, j_{N} \mid}\right|} \int_{\delta_{K, j_{1}, \cdots, j_{N}}} a^{\varepsilon}(x)\left(\nabla \chi_{K, j_{1}, \cdots, j_{N}}^{i, h_{N}}+\mathbf{e}_{i}\right) \cdot\left(\nabla \chi_{K, j_{1}, \cdots, j_{N}}^{k, h_{N}}+\mathbf{e}_{k}\right) d x
\end{aligned}
$$

which also can be written in matrix form as

$a_{K, j_{1}, \cdots, j_{N-1}}^{N-1}\left(x_{K, j_{1}, \cdots, j_{N}}\right)=\frac{1}{\left|\delta_{K, j_{1}, \cdots, j_{N}}\right|} \int_{\delta_{K, j_{1}, \cdots, j_{N}}} a^{\varepsilon}(x)\left(I+J_{\chi_{K, j_{1}, \cdots, j_{N}}^{h_{N}}(x)}^{T}\right) \cdot\left(I+J_{\chi_{K, j_{1}, \cdots, j_{N}}^{h_{N}}}^{T}\right) d x$.

Using the ellipticity of $a^{\varepsilon}(x)$ and (15), we have

$$
\begin{aligned}
\lambda\left\|e_{i}+\nabla \chi_{K, j_{1}, \cdots, j_{N}}^{i, h_{N}}(x)\right\|_{L^{2}\left(\delta_{K, j_{1}, \cdots, j_{N}}\right)}^{2} & \leq \int_{\delta_{K, j_{1}, \cdots, j_{N}}} a^{\varepsilon}(x)\left(\nabla \chi_{K, j_{1}, \cdots, j_{N}}^{i, h_{N}}+\mathbf{e}_{i}\right) \cdot\left(\nabla \chi_{K, j_{1}, j_{2}}^{i, h_{N}}+\mathbf{e}_{i}\right) d x \\
& =\int_{\delta_{K, j_{1}, \cdots, j_{N}}} a^{\varepsilon}(x) \mathbf{e}_{i} \cdot \mathbf{e}_{i}-a^{\varepsilon}(x) \nabla \chi_{K, j_{1}, \cdots, j_{N}}^{i, h_{N}} \cdot \nabla \chi_{K, j_{1}, \cdots, j_{N}}^{i, h_{N}} d x \\
& \leq \Lambda\left|\delta_{K, j_{1}, \cdots, j_{N}}\right| .
\end{aligned}
$$


Therefore we obtain the upper bound. The lower bound is straightforward from the following equality which can be shown by using integration by part and the boundary conditions of the cell problems

$$
\left\|e_{i}+\nabla \chi_{K, j_{1}, \cdots, j_{N}}^{i, h_{N}}(x)\right\|_{L^{2}\left(\delta_{K, j_{1}, \cdots, j_{N}}\right)}^{2}=\left|\delta_{K, j_{1}, \cdots, j_{N}}\right|+\left\|\nabla \chi_{K, j_{1}, \cdots, j_{N}}^{i, h_{N}}(x)\right\|_{L^{2}\left(\delta_{K, j_{1}, \cdots, j_{N}}\right)}^{2} .
$$

We next show the ellipticity of tensor $a_{K, j_{1}, \cdots, j_{N-1}}^{N-1}\left(x_{K, j_{1}, \cdots, j_{N}}\right)$. Using (16) and the symmetry of $a^{\varepsilon}(x)$, we obtain

$$
\begin{aligned}
& a_{K, j_{1}, \cdots, j_{N-1}}^{N-1}\left(x_{K, j_{1}, \cdots, j_{N}}\right) \xi \cdot \xi \\
& =\frac{1}{\left|\delta_{K, j_{1}, \cdots, j_{N}}\right|} \int_{\delta_{K, j_{1}, \cdots, j_{N}}} a^{\varepsilon}(x)\left(( I + J _ { \chi _ { K , j _ { 1 } , \cdots , j _ { N } } } ^ { T } ( x ) \xi ) \cdot \left(\left(I+J_{\chi_{K, j_{1}, \cdots, j_{N}}^{h_{N}}}^{T}(x) \xi\right) d x\right.\right. \\
& \geq \frac{\lambda}{\left|\delta_{K, j_{1}, \cdots, j_{N}}\right|} \int_{\delta_{K, j_{1}, \cdots, j_{N}}}\left(\left(I+J_{\chi_{K, j_{1}, \cdots, j_{N}}}^{T}{ }_{h_{N}}(x) \xi\right) \cdot\left(\left(I+J_{\chi_{K, j_{1}, \cdots, j_{N}}^{h_{N}}(x)}^{T}\right) \xi\right) d x\right. \\
& =\lambda|\xi|^{2}+\frac{\lambda}{\left|\delta_{K, j_{1}, \cdots, j_{N}}\right|} \int_{\delta_{K, j_{1}, \cdots, j_{N}}}\left(J_{\chi_{K, j_{1}, \cdots, j_{N}}}^{T}(x)\right. \\
& \geq \lambda|\xi|^{2} .
\end{aligned}
$$

The second inequality of (14) can be obtained by using (13).

Lemma 4.2. Assume that the hypothesis of Lemma (4.1) holds. Furthermore for each scale $s$ $(s=0, \cdots, N-1)$, we assume that the $Q F$ is chosen such that (Q1) (Q2) hold for $r=q_{s}$ and $\sigma=\max \left(2 q_{s}-2, q_{s}\right)$ if $T_{K, j_{1}, \cdots, j_{s}}$ are simplicial FEs, or $\sigma=\max \left(2 q_{s}-1, q_{s}+1\right)$ if $T_{K, j_{1}, \cdots, j_{s}}$ are rectangular (parallelogram) elements. Then we have

$$
\sqrt{\left|\delta_{K, j_{1}, \cdots, j_{s}}\right|} \leq\left\|\mathbf{e}_{i}+\nabla \chi_{K, j_{1}, \cdots, j_{s}}^{i, h_{s}}\right\|_{L^{2}\left(\delta_{K, j_{1}, \cdots, j_{s}}\right)} \leq C \sqrt{\left|\delta_{K, j_{1}, \cdots, j_{s}}\right|} .
$$

Moreover for $s=1, \cdots, N-1$, we have

$$
a_{K, j_{1}, \cdots, j_{s-1}}^{s-1}\left(x_{K, j_{1}, \cdots, j_{s}}\right) \xi \cdot \xi \geq \lambda|\xi|^{2},\left|a_{K, j_{1}, \cdots, j_{s-1}}^{s-1}\left(x_{K, j_{1}, \cdots, j_{s}}\right) \xi\right| \leq \tilde{C}|\xi|, \quad \forall \xi \in \mathbb{R}^{d},
$$

for any quadrature points $x_{K, j_{1}, \cdots, j_{s}}$ used on the scale $s-1$ and $C, \tilde{C}>1$ depend on $\lambda, \Lambda, d, s, N$. In particular, for $s=1$ we have

$$
a_{K}^{0}\left(x_{K, j_{1}}\right) \xi \cdot \xi \geq \lambda|\xi|^{2},\left|a_{K}^{0}\left(x_{K, j_{1}}\right) \xi\right| \leq \tilde{C}|\xi|, \forall \xi \in \mathbb{R}^{d} .
$$

Proof. The inequality (17) and (18) need to be proved recursively. We assume that (18) holds for $a_{K, j_{1}, \cdots, j_{s}}^{s}\left(x_{K, j_{1}, \cdots, j_{s+1}}\right)$ and first we prove (17), based on which we then prove (18) holding for $a_{K, j_{1}, \cdots, j_{s-1}}^{s-1}\left(x_{K, j_{1}, \cdots, j_{s}}\right)$. We observe that the lower bound of $\left\|\mathbf{e}_{i}+\nabla \chi_{K, j_{1}, \cdots, j_{s}}^{i, h_{s}}(x)\right\|_{L^{2}\left(\delta_{K, j_{1}, \cdots, j_{s}}\right)}$ can be derived similarly to the corresponding steps in Lemma 4.1. For the upper bound, using assumption (Q1), we can write

$$
\begin{aligned}
& \lambda\left\|\nabla \chi_{K, j_{1}, \cdots, j_{s}}^{i, h_{s}}(x)+\mathbf{e}_{i}\right\|_{L^{2}\left(\delta_{K, j_{1}, \cdots, j_{s}}\right)}^{2} \leq \sum_{T_{K, j_{1}, \cdots, j_{s}} \in \mathcal{T}_{K, j_{1}, \cdots, j_{s}}} \sum_{j_{s+1}=1}^{J_{s+1}} \omega_{K, j_{1}, \cdots, j_{s+1}} \cdot \\
& a_{K, j_{1}, \cdots, j_{s}}^{s}\left(x_{K, j_{1}, \cdots, j_{s+1}}\right)\left(\nabla \chi_{K, j_{1}, \cdots, j_{s}}^{i, h_{s}}\left(x_{K, j_{1}, \cdots, j_{s+1}}\right)+\mathbf{e}_{i}\right) \cdot\left(\nabla \chi_{K, j_{1}, \cdots, j_{s}}^{i, h_{s}}\left(x_{K, j_{1}, \cdots, j_{s+1}}\right)+\mathbf{e}_{i}\right) \\
& \leq C\left|\delta_{K, j_{1}, \cdots, j_{s}}\right|,
\end{aligned}
$$


where the last inequality is obtained following the steps of the proof for the upper bound in (13).

In what follows we only prove the first inequlity of (18) (the second inequality can be simply obtained by using the upper bound in inequality (17)). We consider the definition of $a_{K, j_{1}, \cdots, j_{s-1}}^{s-1}\left(x_{K, j_{1}, \cdots, j_{s}}\right)$ in (9) and using (PS),the symmetry of $a_{K, j_{1}, \cdots, j_{s}}^{s}\left(x_{K, j_{1}, \cdots, j_{s+1}}\right)$ and assumption (Q2) we have

$$
\begin{aligned}
a_{K, j_{1}, \cdots, j_{s-1}}^{s-1}\left(x_{K, j_{1}, \cdots, j_{s}}\right) \xi \cdot \xi & \frac{1}{\left|\delta_{K, j_{1}, \cdots, j_{s}}\right|} \sum_{T_{K, j_{1}, \cdots, j_{s}} \in \mathcal{T}_{K, j_{1}, \cdots, j_{s}}} \sum_{j_{s+1}=1}^{J_{s+1}} \omega_{K, j_{1}, \cdots, j_{s+1}} \cdot \\
& a_{K, j_{1}, \cdots, j_{s}}^{s}\left(x_{K, j_{1}, \cdots, j_{s+1}}\right)\left(\left(I+J_{\chi_{K, j_{1}}^{h_{s}}\left(x_{K, j_{1}, \cdots, j_{s}}\right)}^{T}\right) \xi\right) \cdot\left(\left(I+J_{\chi_{K, j_{1}, \cdots, j_{s}}^{h_{s}}\left(x_{K, j_{1}, \cdots, j_{s}}\right)}^{T}\right) \xi\right) \\
\geq & \lambda \frac{1}{\left|\delta_{K, j_{1}, \cdots, j_{s}}\right|} \sum_{T_{K, j_{1}, \cdots, j_{s}} \in \mathcal{T}_{K, j_{1}, \cdots, j_{s}}} \sum_{j_{s+1}=1}^{J_{s+1}} \omega_{K, j_{1}, \cdots, j_{s+1}} \cdot \\
& \left(\left(I+J_{\chi_{K, j_{1}, \cdots, j_{s}}^{h_{s}}\left(x_{K, j_{1}, \cdots, j_{s}}\right)}^{T}\right) \xi\right) \cdot\left(\left(I+J_{\chi_{K, j_{1}, \cdots, j_{s}}^{h_{s}}}^{T}\left(x_{K, j_{1}, \cdots, j_{s}}\right) \xi\right)\right. \\
\geq & \frac{\lambda}{\left|\delta_{K, j_{1}, \cdots, j_{s}}\right|} \int_{\delta_{K, j_{1}, \cdots, j_{s}}}\left(\left(I+J_{\chi_{K, j_{1}, \cdots, j_{s}}^{h_{s}}(x)}^{T}\right) \xi\right) \cdot\left(\left(I+J_{\chi_{K, j_{1}, \cdots, j_{s}}^{h_{s}}(x)}^{T}\right) \xi\right) d x \\
\geq & \lambda|\xi|^{2}+\frac{\lambda}{\left|\delta_{K, j_{1}, \cdots, j_{s}}\right|} \int_{\delta_{K, j_{1}, \cdots, j_{s}}}\left(J_{\chi_{K, j_{1}, \cdots, j_{s}}^{h_{s}}(x)}^{T} \xi\right) \cdot\left(J_{\chi_{K, j_{1}, \cdots, j_{s}}^{h_{s}}(x)}^{T} \xi\right) \\
\geq & \lambda|\xi|^{2} .
\end{aligned}
$$

We note that (19) can be similarly proved using the FE-HMM reformulation (12).

With the help of Lemma 4.1 and 4.2, we obtain the existence and uniqueness of the numerical solution $u^{H}$ of problem (P0).

Theorem 4.3. Assume that the hypothesis of Lemma 4.1 and 4.2 holds, then all the cell problems (PS) for $s=1, \cdots, N-1$ and (PN) have unique solutions. Furthermore, problem (P0) also has a unique solution.

\subsection{Error estimates for the $N+1$ scale FE-HMM.}

Assume $u^{0}$ is the exact homogenized solution of (3) and $a^{0}$ is the exact homogenized tensor. For the error analysis, we need to consider the quantity

$$
r_{H M M}:=\sup _{K \in \mathcal{T}_{H}, x_{K, j_{1}} \in \Omega}\left\|a^{0}\left(x_{K, j_{1}}\right)-a_{K}^{0}\left(x_{K, j_{1}}\right)\right\|_{\mathcal{F}},
$$

where $a_{K}^{0}\left(x_{K, j_{1}}\right)$ is defined in (12) and $\|\cdot\|_{\mathcal{F}}$ is defined as $\|A\|_{\mathcal{F}}=\sqrt{\operatorname{trace}\left(A^{T} A\right)}$. It is also convenient to introduce the FEM with numerical quadrature for the (exact) homogenizated problem (3): find $u^{0, H} \in S_{0}^{\ell}\left(\Omega, \mathcal{T}_{H}\right)$ such that

$$
B_{0, H}\left(u^{0, H}, v^{H}\right)=\int_{\Omega} f v^{H} d x, \forall v^{H} \in S_{0}^{\ell}\left(\Omega, \mathcal{T}_{H}\right),
$$


where

$$
B_{0, H}\left(v^{H}, w^{H}\right):=\sum_{K \in \mathcal{T}_{H}} \sum_{j_{1}=1}^{J_{1}} \omega_{K, j_{1}} a^{0}\left(x_{K, j_{1}}\right) \nabla v^{H}\left(x_{K, j_{1}}\right) \cdot \nabla w^{H}\left(x_{K, j_{1}}\right) d x .
$$

We emphasize that as the homogenized tensor $a^{0}(x)$ is unknown (and depends on $N+1$ scales) the equation (21) cannot be used in practice. It is nevertheless useful for the analysis of the FE-HMM.

Theorem 4.4. Assume that (Q1),(Q2) hold for the macro $Q F$ with $r=\ell$ and $\sigma=\max (2 \ell-$ $2, \ell)$ for simplicial macro elements or $\sigma=\max (2 \ell-1, \ell)$ for rectangular macro elements. Assume that $u^{0} \in H^{\ell+1}(\Omega)$ and that either $a^{0}(x) \in W^{\ell, \infty}(\Omega)$ for the $H^{1}$ norm estimate or $a^{0}(x) \in W^{\ell+1, \infty}(\Omega)$ for the $L^{2}$ norm estimate. Then we have

$$
\begin{aligned}
\left\|u^{0}-u^{H}\right\|_{H^{1}(\Omega)} & \leq C\left(H^{\ell}+r_{H M M}\right), \\
\left\|u^{0}-u^{H}\right\|_{L^{2}(\Omega)} & \leq C\left(H^{\ell+1}+r_{H M M}\right),
\end{aligned}
$$

where the constant $C$ is independent of $H, h_{s}, \varepsilon_{s}$ where $s=1, \cdots, N$.

Proof. We first decompose the error term

$$
\left\|u^{0}-u^{H}\right\| \leq\left\|u^{0}-u^{0, H}\right\|+\left\|u^{0, H}-u^{H}\right\|
$$

where $\|\cdot\|$ stands for the $H^{1}$ norm or $L^{2}$ norm and $u^{0, H}$ is the FE solution of (21). By the standard error analysis (see [12]) of FE method with numerical quadrature, the first term of (25) can be bounded by

$$
\left\|u^{0}-u^{0, H}\right\| \leq C H^{p}
$$

where $p=\ell$ for $H^{1}$ norm and $p=\ell+1$ for $L^{2}$ norm.

Furthermore, by the ellipticity of $a_{K}^{0}$ (Lemma 4.2) and the bound $\left\|\nabla u^{0, H}\right\|_{L^{2}(\Omega)} \leq C\|f\|_{L^{2}(\Omega)}$, using the reformulation (11), we have

$$
\begin{aligned}
\lambda\left\|\nabla u^{0, H}-\nabla u^{H}\right\|_{L^{2}(\Omega)}^{2} & \leq B_{H}\left(u^{0, H}-u^{H}, u^{0, H}-u^{H}\right) \\
& =B_{H}\left(u^{0, H}, u^{0, H}-u^{H}\right)-\int_{\Omega} f\left(u^{0, H}-u^{H}\right) d x \\
& =B_{H}\left(u^{0, H}, u^{0, H}-u^{H}\right)-B_{0, H}\left(u^{0, H}, u^{0, H}-u^{H}\right) \\
& \leq C \sup _{K \in \mathcal{T}_{H}, x_{K, j_{1}} \in \Omega}\left\|a^{0}\left(x_{K, j_{1}}\right)-a_{K}^{0}\left(x_{K, j_{1}}\right)\right\|_{\mathcal{F}}\left\|\nabla u^{0, H}-\nabla u^{H}\right\|_{L^{2}(\Omega)} .
\end{aligned}
$$

Therefore, we obtain

$$
\left\|\nabla u^{0, H}-\nabla u^{H}\right\|_{L^{2}(\Omega)} \leq C r_{H M M} .
$$

Using the last inequality and the Poincaré inequality gives the stated results. 


\section{Estimates for $r_{H M M}$}

The quantity $r_{H M M}$ can be analyzed provided appropriate assumption on the tensor $a^{\varepsilon}$. The contribution to the term $r_{H M M}$ coming from the micro and meso discretization error can in principle be quantified assuming appropriate regularity of $a^{\varepsilon}$. As mentioned in the introduction, to avoid considerable technicality in proofs due to intricate notations, we will restrict to three scale problems and we will indicate the generalization our result to $N+1$ scale problems at the end of this section. We will denote our three scale tensor as $a\left(x, \frac{x}{\varepsilon_{1}}, \frac{x}{\varepsilon_{2}}\right)=$ $a(x, y, z)$ where $y=\frac{x}{\varepsilon_{1}}, z=\frac{x}{\varepsilon_{2}}$ and we will assume periodicity at the meso and micro scales, i.e., assumption (5). For convenience we will denote the assumptions (4),(5) and (6) (for our three scale problem) as assumption (H1).

Under the assumption (H1), as mentioned in Section 2, homogenization results provide an explicit characterization of the tensor $a^{1}(x, y)$ and periodicity with respect to $y$ can be proved. In turn, the exact macro homogenized tensor $a^{0}\left(x_{K, j_{1}}\right)$ at the macro quadrature point $x_{K, j_{1}}$ can be computed as (here we require $\delta_{1} / \varepsilon_{1} \in \mathbb{N}$ )

$$
a^{0}\left(x_{K, j_{1}}\right)=\frac{1}{\left|\delta_{K, j_{1}}\right|} \int_{\delta_{K, j_{1}}} a^{1}\left(x_{K, j_{1}}, \frac{x}{\varepsilon_{1}}\right)\left(I+J_{\chi_{K, j_{1}}^{1}(x)}^{T}\right) d x,
$$

where $\left(J_{\chi_{K, j_{1}}^{1}(x)}^{T}\right)_{i k}=\partial_{k}\left(\chi_{K, j_{1}}^{i, 1}(x)\right)$ and $\chi_{K, j_{1}}^{i, 1}(x) \in W_{p e r}^{1}\left(\delta_{K, j_{1}}\right)$ is the solution of the meso cell problem

$$
\int_{\delta_{K, j_{1}}} a^{1}\left(x_{K, j_{1}}, \frac{x}{\varepsilon_{1}}\right)\left(\nabla \chi_{K, j_{1}}^{i, 1}(x)+\mathbf{e}_{i}\right) \cdot \nabla z d x=0, \quad \forall z \in W_{p e r}^{1}\left(\delta_{K, j_{1}}\right) .
$$

We can also formulate the mesoscopic homogenized tensor $a^{1}\left(x, \frac{x}{\varepsilon_{1}}\right)$ on the couple of quadrature points $\left\{x_{K, j_{1}}, x_{K, j_{1}, j_{2}}\right\}$ as (also assume $\delta_{2} / \varepsilon_{2} \in \mathbb{N}$ )

$$
a^{1}\left(x_{K, j_{1}}, \frac{x_{K, j_{1}, j_{2}}}{\varepsilon_{1}}\right)=\frac{1}{\left|\delta_{K, j_{1}, j_{2}}\right|} \int_{\delta_{K, j_{1}, j_{2}}} a\left(x_{K, j_{1}}, \frac{x_{K, j_{1}, j_{2}}}{\varepsilon_{1}}, \frac{x}{\varepsilon_{2}}\right)\left(I+J_{\chi_{K, j_{1}, j_{2}}^{T}(x)}^{T}\right) d x,
$$

where $\left(J_{\chi_{K, j_{1}, j_{2}}^{2}(x)}^{T}\right)_{i k}=\partial_{k}\left(\chi_{K, j_{1}, j_{2}}^{i, 2}(x)\right)$ and $\chi_{K, j_{1}, j_{2}}^{i, 2}(x) \in W_{p e r}^{1}\left(\delta_{K, j_{1}, j_{2}}\right)$ is the solution of the following micro cell problem

$$
\int_{\delta_{K, j_{1}, j_{2}}} a\left(x_{K, j_{1}}, \frac{x_{K, j_{1}, j_{2}}}{\varepsilon_{1}}, \frac{x}{\varepsilon_{2}}\right)\left(\nabla \chi_{K, j_{1}, j_{2}}^{i, 2}(x)+\mathbf{e}_{i}\right) \cdot \nabla z d x=0, \quad \forall z \in W_{p e r}^{1}\left(\delta_{K, j_{1}, j_{2}}\right) .
$$

As discussed in [4], appropriate regularity assumptions are required for the functions $\chi_{K, j_{1}}^{i, 1}$ and $\chi_{K, j_{1}, j_{2}}^{i, 2}$ defined in (27) and (29), respectively. We assume

(H2) for given positive integers $q_{1}, q_{2}$, the meso and micro cell solutions $\chi_{K, j_{1}}^{i, 1}$ and $\chi_{K, j_{1}, j_{2}}^{i, 2}$ satisfy

$$
\left|\chi_{K, j_{1}}^{i, 1}\right|_{H^{q_{1}+1}\left(\delta_{K, j_{1}}\right)} \leq C \varepsilon_{1}^{-q_{1}} \sqrt{\left|\delta_{K, j_{1}}\right|}, \quad\left|\chi_{K, j_{1}, j_{2}}^{i, 2}\right|_{H^{q_{2}+1}\left(\delta_{K, j_{1}, j_{2}}\right)} \leq C \varepsilon_{2}^{-q_{2}} \sqrt{\left|\delta_{K, j_{1}, j_{2}}\right|} .
$$

Remark 5.1. Similarly to (17) and (13), one can show (without using (H2))

$$
\left\|\nabla \chi_{K, j_{1}}^{i, 1}+\mathbf{e}_{i}\right\|_{L^{2}\left(\delta_{K, j_{1}}\right)} \leq C \sqrt{\left|\delta_{K, j_{1}}\right|}, \quad\left\|\nabla \chi_{K, j_{1}, j_{2}}^{i, 2}+\mathbf{e}_{i}\right\|_{L^{2}\left(\delta_{K, j_{1}, j_{2}}\right)} \leq C \sqrt{\left|\delta_{K, j_{1}, j_{2}}\right|},
$$

where the constant $C$ only depends on $\lambda$ and $\Lambda$ introduced in (2). 
In this subsection, we consider that the meso FE space $S^{q_{1}}\left(\delta_{K, j_{1}}, \mathcal{T}_{K, j_{1}}\right)$ and the micro FE space $S^{q_{2}}\left(\delta_{K, j_{1}, j_{2}}, \mathcal{T}_{K, j_{1}, j_{2}}\right)$ are the subspace of $W_{p e r}\left(\delta_{K, j_{1}}\right), W_{p e r}\left(\delta_{K, j_{1}, j_{2}}\right)$ respectively or of $H_{0}^{1}\left(\delta_{K, j_{1}}\right), H_{0}^{1}\left(\delta_{K, j_{1}, j_{2}}\right)$ (see $(7)$ and $\left.(8)\right)$. In order to distinguish the FE spaces with different boundary conditions, we denote the meso and micro FE spaces by $S_{0}^{q_{1}}\left(\delta_{K, j_{1}}, \mathcal{T}_{K, j_{1}}\right)$ and $S_{0}^{q_{2}}\left(\delta_{K, j_{1}, j_{2}}, \mathcal{T}_{K, j_{1}, j_{2}}\right)$ for the Dirchlet boundary coupling and keep the notations $S^{q_{1}}\left(\delta_{K, j_{1}}, \mathcal{T}_{K, j_{1}}\right)$, $S^{q_{2}}\left(\delta_{K, j_{1}, j_{2}}, \mathcal{T}_{K, j_{1}, j_{2}}\right)$ for periodic boundary coupling.

Before starting the analysis, we need to define several notations. Denote $\bar{\chi}_{K, j_{1}}^{i, h_{1}}(x) \in$ $S^{q_{1}}\left(\delta_{K, j_{1}}, \mathcal{T}_{K, j_{1}}\right)$ or $S_{0}^{q_{1}}\left(\delta_{K, j_{1}}, \mathcal{T}_{K, j_{1}}\right)$ the solution of

$$
\sum_{T_{K, j_{1}} \in \mathcal{T}_{K, j_{1}}} \sum_{j_{2}=1}^{J_{2}} \omega_{K, j_{1}, j_{2}} a^{1}\left(x_{K, j_{1}}, \frac{x_{K, j_{1}, j_{2}}}{\varepsilon_{1}}\right)\left(\nabla \bar{\chi}_{K, j_{1}}^{i, h_{1}}\left(x_{K, j_{1}, j_{2}}\right)+\mathbf{e}_{i}\right) \cdot \nabla z^{h_{1}}=0,
$$

where $z^{h_{1}}$ is an arbitrary function in $S^{q_{1}}\left(\delta_{K, j_{1}}, \mathcal{T}_{K, j_{1}}\right)$ or $S_{0}^{q_{1}}\left(\delta_{K, j_{1}}, \mathcal{T}_{K, j_{1}}\right)$ correspondingly, and define the tensor $\bar{a}_{K}^{0}\left(x_{K, j_{1}}\right)$ as

$$
\bar{a}_{K}^{0}\left(x_{K, j_{1}}\right)=\frac{1}{\left|\delta_{K, j_{1}}\right|} \sum_{T_{K, j_{1}} \in \mathcal{T}_{K, j_{1}}} \sum_{j_{2}=1}^{J_{2}} \omega_{K, j_{1}, j_{2}} a^{1}\left(x_{K, j_{1}}, \frac{x_{K, j_{1}, j_{2}}}{\varepsilon_{1}}\right)\left(I+J_{\bar{\chi}_{K, j_{1}}^{h_{1}}\left(x_{K, j_{1}, j_{2}}\right)}^{T}\right) .
$$

Likewise, we consider $\bar{\chi}_{K, j_{1}, j_{2}}^{i, h_{2}}(x) \in S^{q_{2}}\left(\delta_{K, j_{1}, j_{2}}, \mathcal{T}_{K, j_{1}, j_{2}}\right)$ or $S_{0}^{q_{2}}\left(\delta_{K, j_{1}, j_{2}}, \mathcal{T}_{K, j_{1}, j_{2}}\right)$ be the solution of

$$
\int_{\delta_{K, j_{1}, j_{2}}} a\left(x_{K, j_{1}}, \frac{x_{K, j_{1}, j_{2}}}{\varepsilon_{1}}, \frac{x}{\varepsilon_{2}}\right)\left(\nabla \bar{\chi}_{K, j_{1}, j_{2}}^{i, h_{2}}(x)+\mathbf{e}_{i}\right) \cdot \nabla z^{h_{2}} d x=0
$$

and define

$$
\bar{a}_{K, j_{1}}^{1}\left(x_{K, j_{1}, j_{2}}\right)=\frac{1}{\left|\delta_{K, j_{1}, j_{2}}\right|} \int_{\delta_{K, j_{1}, j_{2}}} a\left(x_{K, j_{1}}, \frac{x_{K, j_{1}, j_{2}}}{\varepsilon_{1}}, \frac{x}{\varepsilon_{2}}\right)\left(I+J_{\bar{\chi}_{K, j_{1}, j_{2}}^{h_{2}}(x)}\right) d x .
$$

Remark 5.2. Using the uniform boundedness and ellipticity of the tensor a $(x, y, z)$ (part of the assumption in (H1)), one can show that $a^{1}(x, y), \bar{a}_{K, j_{1}}^{1}(x)$ are also elliptic and bounded. In turn, the boundedness and ellipticity of $a^{0}\left(x_{K, j_{1}}\right), \bar{a}_{K}^{0}\left(x_{K, j_{1}}\right)$ can be established.

Lemma 5.3. Assume (H1), (H2) and that $a^{\varepsilon}$ is symmetric, uniformly bounded and elliptic. Furthermore we consider the meso and micro FE spaces are $S^{q_{1}}\left(\delta_{K, j_{1}}, \mathcal{T}_{K, j_{1}}\right), S^{q_{2}}\left(\delta_{K, j_{1}, j_{2}}, \mathcal{T}_{K, j_{1}, j_{2}}\right)$ and $\delta_{1} / \varepsilon_{1} \in \mathbb{N}, \delta_{2} / \varepsilon_{2} \in \mathbb{N}$. Then we have

$$
\left\|a^{1}\left(x_{K, j_{1}}, \frac{x_{K, j_{1}, j_{2}}}{\varepsilon_{1}}\right)-\bar{a}_{K, j_{1}}^{1}\left(x_{K, j_{1}, j_{2}}\right)\right\|_{\mathcal{F}} \leq C\left(\frac{h_{2}}{\varepsilon_{2}}\right)^{2 q_{2}} .
$$

In addition, we assume that $a^{1}(\cdot, y) \in W^{2 q_{1}}(Y)$ and that assumption (Q2) holds for the mesoscopic QF with $r=q_{1}$ and $\sigma=\max \left(2 q_{1}-1, q_{1}\right)$ if $T_{K, j_{1}}$ are simplicial FEs, or $\sigma=$ $\max \left(2 q_{1}-1, q_{1}+1\right)$ if $T_{K, j_{1}}$ are rectangular (parallelogram) FEs. Then we can show

$$
\left\|a^{0}\left(x_{K, j_{1}}\right)-\bar{a}_{K}^{0}\left(x_{K, j_{1}}\right)\right\|_{\mathcal{F}} \leq C\left(\frac{h_{1}}{\varepsilon_{1}}\right)^{2 q_{1}} .
$$


Proof. The proof of (35) can be obtained by following the corresponding proof for two scale problems. The detail can be seen in $[1,3]$ and we skip this part here.

Next, we show (36). We define an auxiliary tensor

$$
\hat{a}_{K}^{0}\left(x_{K, j_{1}}\right) \mathbf{e}_{i} \cdot \mathbf{e}_{k}=\frac{1}{\left|\delta_{K, j_{1}}\right|} \int_{\delta_{K, j_{1}}} a^{1}\left(x_{K, j_{1}}, \frac{x}{\varepsilon_{1}}\right)\left(\nabla \bar{\chi}_{K, j_{1}}^{i, h_{1}}(x)+\mathbf{e}_{i}\right) \cdot\left(\nabla \bar{\chi}_{K, j_{1}}^{k, h_{1}}(x)+\mathbf{e}_{k}\right) d x .
$$

Then we consider the decomposition

$$
\left\|a^{0}\left(x_{K, j_{1}}\right)-\bar{a}_{K}^{0}\left(x_{K, j_{1}}\right)\right\|_{\mathcal{F}} \leq\left\|a^{0}\left(x_{K, j_{1}}\right)-\hat{a}_{K}^{0}\left(x_{K, j_{1}}\right)\right\|_{\mathcal{F}}+\left\|\hat{a}^{0}\left(x_{K, j_{1}}\right)-\bar{a}_{K}^{0}\left(x_{K, j_{1}}\right)\right\|_{\mathcal{F}} .
$$

Using (27) and the symmetry of $a^{1}(x, y)$, we have

$$
\begin{aligned}
& \left(a^{0}\left(x_{K, j_{1}}\right)-\hat{a}_{K}^{0}\left(x_{K, j_{1}}\right)\right) \mathbf{e}_{i} \cdot \mathbf{e}_{k} \\
& =\frac{1}{\left|\delta_{K, j_{1}}\right|} \int_{\delta_{K, j_{1}}} a^{1}\left(x_{K, j_{1}}, \frac{x}{\varepsilon_{1}}\right)\left(\nabla \chi_{K, j_{1}}^{i, 1}(x)-\nabla \bar{\chi}_{K, j_{1}}^{i, h_{1}}(x)\right) \cdot\left(\nabla \chi_{K, j_{1}}^{k, 1}(x)+\mathbf{e}_{k}\right) d x \\
& +\frac{1}{\left|\delta_{K, j_{1}}\right|} \int_{\delta_{K, j_{1}}} a^{1}\left(x_{K, j_{1}}, \frac{x}{\varepsilon_{1}}\right)\left(\nabla \bar{\chi}_{K, j_{1}}^{i, h_{1}}(x)+\mathbf{e}_{i}\right) \cdot\left(\nabla \chi_{K, j_{1}}^{k, 1}(x)-\nabla \bar{\chi}_{K, j_{1}}^{k, h_{1}}(x)\right) d x \\
& =\frac{1}{\left|\delta_{K, j_{1}}\right|} \int_{\delta_{K, j_{1}}} a^{1}\left(x_{K, j_{1}}, \frac{x}{\varepsilon_{1}}\right)\left(\nabla \bar{\chi}_{K, j_{1}}^{i, h_{1}}(x)-\nabla \chi_{K, j_{1}}^{i, 1}(x)\right) \cdot\left(\nabla \chi_{K, j_{1}}^{k, 1}(x)-\nabla \bar{\chi}_{K, j_{1}}^{k, h_{1}}(x)\right) d x .
\end{aligned}
$$

Applying Cauchy-Schwarz inequality, the standard FE a priori error estimate for FEM with numerical quadrature gives $\left\|\nabla \chi_{K, j_{1}}^{i, 1}(x)-\nabla \bar{\chi}_{K, j_{1}}^{i, h_{1}}(x)\right\|_{L^{2}\left(\delta_{K, j_{1}}\right)} \leq C h_{1}^{q_{1}}\left|\chi_{K, j_{1}}^{i, 1}(x)\right|_{H^{q_{1}+1}\left(\delta_{K, j_{1}}\right)}$ and using assumption (H2), we obtain

$$
\left\|a^{0}\left(x_{K, j_{1}}\right)-\hat{a}_{K}^{0}\left(x_{K, j_{1}}\right)\right\|_{\mathcal{F}} \leq C\left(\frac{h_{1}}{\varepsilon_{1}}\right)^{2 q_{1}} .
$$

For the second term of (37), an application of Bramble-Hilbert theorem (see [10] and the appendix) using the regularity assumption $a^{1}(\cdot, y) \in W^{2 q_{1}}(Y)$, the assumption (Q2) on mesoscopic QF and the assumption (H2) gives

$$
\left\|\hat{a}_{K}^{0}\left(x_{K, j_{1}}\right)-\bar{a}^{0}\left(x_{K, j_{1}}\right)\right\|_{\mathcal{F}} \leq C\left(\frac{h_{1}}{\varepsilon_{1}}\right)^{2 q_{1}},
$$

and the proof of (36) is complete.

In order to address a corresponding lemma for Dirichlet boundary coupling, we first define $\xi_{K, j_{1}}^{i, 1}(x) \in H_{0}^{1}\left(\delta_{K, j_{1}}\right)$ the solution of (27) with test function in $H_{0}^{1}\left(\delta_{K, j_{1}}\right)$ (note that for Dirichlet boundary coupling we neither assume $\delta_{1} / \varepsilon_{1} \in \mathbb{N}$ nor $\left.\delta_{2} / \varepsilon_{2} \in \mathbb{N}\right)$. Similarly we define $\xi_{K, j_{1}, j_{2}}^{i, 2}(x) \in H_{0}^{1}\left(\delta_{K, j_{1}, j_{2}}\right)$ the solution of $(29)$ with test function in $H_{0}^{1}\left(\delta_{K, j_{1}, j_{2}}\right)$.

Lemma 5.4. Let $W\left(\delta_{K, j_{1}}\right)=H_{0}^{1}\left(\delta_{K, j_{1}}\right)$ and $W\left(\delta_{K, j_{1}, j_{2}}\right)=H_{0}^{1}\left(\delta_{K, j_{1}, j_{2}}\right)$ for the meso and micro cell problems of the FE-HMM respectively where $\delta_{1}>\varepsilon_{1}, \delta_{2}>\varepsilon_{2}$. Assume (H1), (H2), and the mesoscopic QF satisfies (Q2) with $r=q_{1}$ and $\sigma=\max \left(2 q_{1}-1, q_{1}\right)$ if $T_{K, j_{1}}$ are simplicial FEs, or $\sigma=\max \left(2 q_{1}-1, q_{1}+1\right)$ if $T_{K, j_{1}}$ are rectangular (parallelogram) $F E s$ elements. Further assume the following additional regularity assumption on the cell problems,

(H3) for each meso and micro sampling domain $\delta_{K, j_{1}}$ and $\delta_{K, j_{1}, j_{2}}$, assume $\xi_{K, j_{1}}^{i, 1}(x), \chi_{K, j_{1}}^{i, 1}(x) \in$ 
$W^{1, \infty}\left(\delta_{K, j_{1}}\right)$ and $\xi_{K, j_{1}, j_{2}}^{i, 2}(x), \chi_{K, j_{1}, j_{2}}^{i, 2}(x) \in W^{1, \infty}\left(\delta_{K, j_{1}, j_{2}}\right), i=1, \cdots, d$, where $\xi_{K, j_{1}}^{i, 1}(x), \xi_{K, j_{1}, j_{2}}^{i, 2}(x)$ are defined above and $\chi_{K, j_{1}}^{i, 1}(x), \chi_{K, j_{1}, j_{2}}^{i, 2}(x)$ are defined in (27) and (29), respectively.

Then we have

$$
\begin{aligned}
& \left\|a^{0}\left(x_{K, j_{1}}\right)-\bar{a}_{K}^{0}\left(x_{K, j_{1}}\right)\right\|_{\mathcal{F}} \leq C\left(\left(\frac{h_{1}}{\varepsilon_{1}}\right)^{2 q_{1}}+\frac{\varepsilon_{1}}{\delta_{1}}\right), \\
& \left\|a^{1}\left(x_{K, j_{1}}, \frac{x_{K, j_{1}, j_{2}}}{\varepsilon_{1}}\right)-\bar{a}_{K, j_{1}}^{1}\left(x_{K, j_{1}, j_{2}}\right)\right\|_{\mathcal{F}} \leq C\left(\left(\frac{h_{2}}{\varepsilon_{2}}\right)^{2 q_{2}}+\frac{\varepsilon_{2}}{\delta_{2}}\right) .
\end{aligned}
$$

Proof. We only give the proof for (38) here as (39) can be proved similarly. Here we use the following expression for $a^{0}\left(x_{K, j_{1}}\right)$ (see $(26)$ ),

$$
a^{0}\left(x_{K, j_{1}}\right)=\frac{1}{\left|\varepsilon_{K, j_{1}}\right|} \int_{\varepsilon_{K, j_{1}}} a^{1}\left(x_{K, j_{1}}, \frac{x}{\varepsilon_{1}}\right)\left(I+J_{\chi_{K, j_{1}}^{1}(x)}^{T}\right) d x,
$$

where $\varepsilon_{K, j_{1}}$ a meso sampling domain centered at $x_{K, j_{1}}$ which covers the maximum number of $\varepsilon_{1}$ period in each direction contained in the domain $\delta_{K, j_{1}}$, i.e. $\left|\varepsilon_{K, j_{1}}\right|=\left(N \varepsilon_{1}\right)^{d}, N \in \mathbb{N}$ (note that $\delta_{1} / \varepsilon_{1}$ may not belong to $\mathbb{N}$ ). We define the following tensor

$$
\bar{a}^{0}\left(x_{K, j_{1}}\right)=\frac{1}{\left|\delta_{K, j_{1}}\right|} \int_{\delta_{K, j_{1}}} a^{1}\left(x_{K, j_{1}}, \frac{x}{\varepsilon_{1}}\right)\left(I+J_{\xi_{K, j_{1}}^{1}(x)}^{T}\right) d x
$$

based on the cell functions $\xi_{K, j_{1}}^{i, 1}(x)$ defined above and further decompose

$$
\left\|a^{0}\left(x_{K, j_{1}}\right)-\bar{a}_{K}^{0}\left(x_{K, j_{1}}\right)\right\|_{\mathcal{F}} \leq\left\|a^{0}\left(x_{K, j_{1}}\right)-\bar{a}^{0}\left(x_{K, j_{1}}\right)\right\|_{\mathcal{F}}+\left\|\bar{a}^{0}\left(x_{K, j_{1}}\right)-\bar{a}_{K}^{0}\left(x_{K, j_{1}}\right)\right\|_{\mathcal{F}},
$$

where $\bar{a}_{K}^{0}\left(x_{K, j_{1}}\right)$ is defined in (32). Similarly to the proof of Lemma 5.3, one can deduce that the second term of (42) can be bounded as

$$
\left\|\bar{a}^{0}\left(x_{K, j_{1}}\right)-\bar{a}_{K}^{0}\left(x_{K, j_{1}}\right)\right\|_{\mathcal{F}} \leq C\left(\frac{h_{1}}{\varepsilon_{1}}\right)^{2 q_{1}} .
$$

For the first term of (42), one needs to apply a boundary corrector technique to obtain the error due to the nonconformity of the boundary condition of the cell problem. This has first been studied in [17] for the FE-HMM. We give here a short proof for completeness.

We first write

$$
\begin{aligned}
& \left|\left(a^{0}\left(x_{K, j_{1}}\right)-\bar{a}^{0}\left(x_{K, j_{1}}\right)\right) \mathbf{e}_{i} \cdot \mathbf{e}_{k}\right| \\
& \leq\left|\frac{1}{\left|\delta_{K, j_{1}}\right|} \int_{\delta_{K, j_{1}}} a^{1}\left(x_{K, j_{1}}, \frac{x}{\varepsilon_{1}}\right)\left(\nabla \chi_{K, j_{1}}^{i, 1}(x)+\mathbf{e}_{i}\right) \cdot\left(\nabla \xi_{K, j_{1}}^{k, 1}(x)+\mathbf{e}_{k}\right) d x-\bar{a}^{0}\left(x_{K, j_{1}}\right) \mathbf{e}_{i} \cdot \mathbf{e}_{k}\right| \\
& +\left|a^{0}\left(x_{K, j_{1}}\right) \mathbf{e}_{i} \cdot \mathbf{e}_{k}-\frac{1}{\left|\delta_{K, j_{1}}\right|} \int_{\delta_{K, j_{1}}} a^{1}\left(x_{K, j_{1}}, \frac{x}{\varepsilon_{1}}\right)\left(\nabla \chi_{K, j_{1}}^{i, 1}(x)+\mathbf{e}_{i}\right) \cdot\left(\nabla \xi_{K, j_{1}}^{k, 1}(x)+\mathbf{e}_{k}\right) d x\right| \\
& :=I+I I .
\end{aligned}
$$

Let $\Gamma_{K, j_{1}}=\delta_{K, j_{1}} \backslash \varepsilon_{K, j_{1}}$ be the boundary layer where $\left|\Gamma_{K, j_{1}}\right|=C \delta_{1}^{d-1} \varepsilon_{1}$ and $\frac{\left|\Gamma_{K, j_{1}}\right|}{\left|\delta_{K, j_{1}}\right|} \leq$ $C \varepsilon_{1} / \delta_{1}$. By assumption (H3), one can derive

$$
\left\|\nabla \chi_{K, j_{1}}^{i, 1}(x)+\mathbf{e}_{i}\right\|_{L^{2}\left(\Gamma_{K, j_{1}}\right)} \leq C \sqrt{\left|\Gamma_{K, j_{1}}\right|}, \quad\left\|\nabla \xi_{K, j_{1}}^{k, 1}(x)+\mathbf{e}_{k}\right\|_{L^{2}\left(\Gamma_{K, j_{1}}\right)} \leq C \sqrt{\left|\Gamma_{K, j_{1}}\right|} .
$$


We next define function $\rho_{K, j_{1}}^{\varepsilon_{1}} \in \mathcal{C}^{\infty}\left(\delta_{K, j_{1}}\right) ; 0 \leq \rho_{K, j_{1}}^{\varepsilon_{1}} \leq 1$ with the following properties

$$
\rho_{K, j_{1}}^{\varepsilon_{1}}(x)=\left\{\begin{array}{cc}
1 & \operatorname{dist}\left(x, \partial \delta_{K, j_{1}}\right)>2 \varepsilon_{1} \\
0 & \operatorname{dist}\left(x, \partial \delta_{K, j_{1}}\right)<\varepsilon_{1}
\end{array}\right.
$$

and $\varepsilon_{1}\left\|\nabla \rho_{K, j_{1}}^{\varepsilon_{1}}\right\|_{L^{\infty}\left(\delta_{K, j_{1}}\right)} \leq C$ where $C$ is independent of $\varepsilon_{1}$.

We then introduce the boundary corrector $\theta_{K, j_{1}}^{i, 1}:=\xi_{K, j_{1}}^{i, 1}-\chi_{K, j_{1}}^{i, 1}$ which satisfies $\theta_{K, j_{1}}^{i, 1}+$ $\left(1-\rho_{K, j_{1}}^{\varepsilon_{1}}\right) \chi_{K, j_{1}}^{i, 1} \in H_{0}^{1}\left(\delta_{K, j_{1}}\right)$. Thus, by noticing that

$$
\bar{a}^{0}\left(x_{K, j_{1}}\right) \mathbf{e}_{i} \cdot \mathbf{e}_{k}=\frac{1}{\left|\delta_{K, j_{1}}\right|} \int_{\delta_{K, j_{1}}} a^{1}\left(x_{K, j_{1}}, \frac{x}{\varepsilon_{1}}\right)\left(\xi_{K, j_{1}}^{i, 1}(x)+\mathbf{e}_{i}\right) \cdot\left(\xi_{K, j_{1}}^{k, 1}(x)+\mathbf{e}_{k}\right) d x,
$$

we have

$$
\begin{aligned}
I & \leq \frac{1}{\left|\delta_{K, j_{1}}\right|}\left|\int_{\delta_{K, j_{1}}} a^{1}\left(x_{K, j_{1}}, \frac{x}{\varepsilon_{1}}\right) \nabla\left(\theta_{K, j_{1}}^{i, 1}+\chi_{K, j_{1}}^{i, 1}\left(1-\rho_{K, j_{1}}^{\varepsilon_{1}}\right)\right) \cdot\left(\nabla \xi_{K, j_{1}}^{k, 1}+\mathbf{e}_{k}\right) d x\right| \\
& +\frac{1}{\left|\delta_{K, j_{1}}\right|}\left|\int_{\delta_{K, j_{1}}} a^{1}\left(x_{K, j_{1}}, \frac{x}{\varepsilon_{1}}\right) \nabla\left(\chi_{K, j_{1}}^{i, 1}\left(1-\rho_{K, j_{1}}^{\varepsilon_{1}}\right)\right) \cdot\left(\nabla \xi_{K, j_{1}}^{k, 1}+\mathbf{e}_{k}\right) d x\right| .
\end{aligned}
$$

The first term on the right-hand side of the above inequality above vanishes since $\theta_{K, j_{1}}^{i, 1}+$ $\chi_{K, j_{1}}^{i, 1}\left(1-\rho_{K, j_{1}}^{\varepsilon_{1}}\right) \in H_{0}^{1}\left(\delta_{K, j_{1}}\right), a^{1}\left(x_{K, j_{1}}, \frac{x}{\varepsilon_{1}}\right)$ is symmetric and $\xi_{K, j_{1}}^{k, 1}$ is the solution of $(27)$ in $H_{0}^{1}\left(\delta_{K, j_{1}}\right)$. Then we have

$$
\begin{aligned}
I & \leq \frac{1}{\left|\delta_{K, j_{1}}\right|}\left|\int_{\delta_{K, j_{1}}} a^{1}\left(x_{K, j_{1}}, \frac{x}{\varepsilon_{1}}\right)\left(\nabla \chi_{K, j_{1}}^{i, 1}\left(1-\rho_{K, j_{1}}^{\varepsilon_{1}}\right)\right) \cdot\left(\nabla \xi_{K, j_{1}}^{k, 1}+\mathbf{e}_{k}\right) d x\right| \\
& +\frac{1}{\left|\delta_{K, j_{1}}\right|}\left|\int_{\delta_{K, j_{1}}} a^{1}\left(x_{K, j_{1}}, \frac{x}{\varepsilon_{1}}\right)\left(\chi_{K, j_{1}}^{i, 1} \nabla \rho_{K, j_{1}}^{\varepsilon_{1}}\right) \cdot\left(\nabla \xi_{K, j_{1}}^{k, 1}+\mathbf{e}_{k}\right) d x\right| \\
& \leq C \frac{1}{\left|\delta_{K, j_{1}}\right|}\left\|\nabla \xi_{K, j_{1}}^{k, 1}+\mathbf{e}_{k}\right\|_{L^{2}\left(\Gamma_{K, j_{1}}\right)}\left(\left\|\nabla \chi_{K, j_{1}}^{i, 1}\right\|_{L^{2}\left(\Gamma_{K, j_{1}}\right)}+\frac{1}{\varepsilon_{1}}\left\|\chi_{K, j_{1}}^{i, 1}\right\|_{L^{2}\left(\Gamma_{K, j_{1}}\right)}\right) \\
& \leq C \frac{\varepsilon_{1}}{\delta_{1}},
\end{aligned}
$$

where the last inequality is obtained by (44) and the fact that $\chi_{K, j_{1}}^{i, 1}(x)=\varepsilon_{1} \hat{\chi}_{K, j_{1}}^{i, 1}\left(\frac{x}{\varepsilon_{1}}\right)$ where $\hat{\chi}_{K, j_{1}}^{i, 1}(y)$ is the solution of (27) on the reference meso cell $Y$ obtained by the affine mapping from $\delta_{K, j_{1}}$ to $Y$ (observe that $\left\|\hat{\chi}_{K, j_{1}}^{i, 1}\right\|_{L^{2}(Y)} \leq C$ ).

For term $I I$, we have

$$
\begin{aligned}
I I & \leq \frac{\left|\delta_{K, j_{1}}\right|-\left|\varepsilon_{K, j_{1}}\right|}{\left|\delta_{K, j_{1}}\right|} \frac{1}{\left|\varepsilon_{K, j_{1}}\right|}\left|\int_{\varepsilon_{K, j_{1}}} a^{1}\left(x_{K, j_{1}}, \frac{x}{\varepsilon_{1}}\right)\left(\nabla \chi_{K, j_{1}}^{i, 1}(x)+\mathbf{e}_{i}\right) \cdot \nabla \theta_{K, j_{1}}^{k, 1} d x\right| \\
& +\frac{1}{\left|\delta_{K, j_{1}}\right|}\left|\int_{\Gamma_{K, j_{1}}} a^{1}\left(x_{K, j_{1}}, \frac{x}{\varepsilon_{1}}\right)\left(\nabla \chi_{K, j_{1}}^{i, 1}(x)+\mathbf{e}_{i}\right) \cdot\left(\nabla \xi_{K, j_{1}}^{k, 1}(x)+\mathbf{e}_{k}\right) d x\right| \\
& :=(a)+(b) .
\end{aligned}
$$

Finally we have

$$
\begin{aligned}
& (a) \leq C \frac{\left|\delta_{K, j_{1}}\right|-\left|\varepsilon_{K, j_{1}}\right|}{\left|\delta_{K, j_{1}}\right|} \frac{1}{\left|\varepsilon_{K, j_{1}}\right|}\left\|\nabla \chi_{K, j_{1}}^{i, 1}+\mathbf{e}_{i}\right\|_{L^{2}\left(\varepsilon_{K, j_{1}}\right)}\left\|\nabla \theta_{K, j_{1}}^{k, 1}\right\|_{L^{2}\left(\varepsilon_{K, j_{1}}\right)} \leq C \frac{\varepsilon_{1}}{\delta_{1}}, \\
& (b) \leq C \frac{1}{\left|\delta_{K, j_{1}}\right|}\left|\Gamma_{K, j_{1}}\right| \leq C \frac{\varepsilon_{1}}{\delta_{1}} .
\end{aligned}
$$

Therefore, we have shown $\left\|a^{0}\left(x_{K, j_{1}}\right)-\bar{a}^{0}\left(x_{K, j_{1}}\right)\right\|_{\mathcal{F}} \leq C \frac{\varepsilon_{1}}{\delta_{1}}$. 
With the help of Lemma 5.3 and 5.4, we are able to state our main theorem.

Theorem 5.5. Assume (H1), (H2) and that the mesoscopic QF satisfies assumption (Q2) with $r=q_{1}$ and $\sigma=\max \left(2 q_{1}-1, q_{1}\right)$ if $T_{K, j_{1}}$ are simplicial $F E s$, or $\sigma=\max \left(2 q_{1}-1, q_{1}+1\right)$ if $T_{K, j_{1}}$ are rectangular (parallelogram) FEs. Assume that $a^{\varepsilon}$ is symmetric, uniformly bounded and elliptic. Further assume that $a(x, y, z)$ is Lipschitz continuous on $\bar{\Omega} \times \bar{Y} \times \bar{Z}$. Then

$$
r_{H M M} \leq C\left(\left(\frac{h_{1}}{\varepsilon_{1}}\right)^{2 q_{1}}+\left(\frac{h_{2}}{\varepsilon_{2}}\right)^{2 q_{2}}+r_{M O D}\right),
$$

where $r_{M O D}$ stands for the HMM modeling error which is estimated as follows.

- If $W\left(\delta_{K, j_{1}}\right)=W_{p e r}^{1}\left(\delta_{K, j_{1}}\right)$ and $W\left(\delta_{K, j_{1}, j_{2}}\right)=W_{p e r}^{1}\left(\delta_{K, j_{1}, j_{2}}\right)$ with $\delta_{1} / \varepsilon_{1} \in \mathbb{N}, \delta_{2} / \varepsilon_{2} \in \mathbb{N}$, then

$$
r_{M O D} \leq C\left(\delta_{1}+\frac{\delta_{2}}{\varepsilon_{1}}\right)
$$

If in addition, the tensor $a\left(x, \frac{x}{\varepsilon_{1}}, \frac{x}{\varepsilon_{2}}\right)$ in the micro cell problem (PN) for $N=2$ is collocated at the couple of quadrature points $\left\{x_{K, j_{1}}, x_{K, j_{1}, j_{2}}\right\}$ i.e. $a\left(x_{K, j_{1}}, \frac{x_{K, j_{1}, j_{2}}}{\varepsilon_{1}}, \frac{x}{\varepsilon_{2}}\right)$, then

$$
r_{M O D}=0
$$

- If $W\left(\delta_{K, j_{1}}\right)=H_{0}^{1}\left(\delta_{K, j_{1}}\right)$ and $W\left(\delta_{K, j_{1}, j_{2}}\right)=H_{0}^{1}\left(\delta_{K, j_{1}, j_{2}}\right)$ with $\delta_{1}>\varepsilon_{1}, \delta_{2}>\varepsilon_{2}$ and assume (H3) holds then

$$
r_{M O D} \leq C\left(\delta_{1}+\frac{\varepsilon_{1}}{\delta_{1}}+\frac{\delta_{2}}{\varepsilon_{1}}+\frac{\varepsilon_{2}}{\delta_{2}}\right) .
$$

If in addition, the tensor $a\left(x, \frac{x}{\varepsilon_{1}}, \frac{x}{\varepsilon_{2}}\right)$ in the micro cell problem $(\mathbf{P N})$ for $N=2$ is collocated at the couple of quadrature points $\left\{x_{K, j_{1}}, x_{K, j_{1}, j_{2}}\right\}$ i.e. $a\left(x_{K, j_{1}}, \frac{x_{K, j_{1}, j_{2}}}{\varepsilon_{1}}, \frac{x}{\varepsilon_{2}}\right)$, then

$$
r_{M O D} \leq C\left(\frac{\varepsilon_{1}}{\delta_{1}}+\frac{\varepsilon_{2}}{\delta_{2}}\right) .
$$

We will use the following lemma in the proof of Theorem 5.5.

Lemma 5.6. Assume (H2) holds, then we have the following upper bounds for $\bar{\chi}_{K, j_{1}}^{i, h_{1}}$ (defined in (31)) and $\bar{\chi}_{K, j_{1}, j_{2}}^{i, h_{2}}$ (defined in (33))

$$
\begin{aligned}
& \left\|\nabla \bar{\chi}_{K, j_{1}}^{i, h_{1}}+\mathbf{e}_{i}\right\|_{L^{2}\left(\delta_{K, j_{1}}\right)} \leq C \sqrt{\left|\delta_{K, j_{1}}\right|} \\
& \left\|\nabla \bar{\chi}_{K, j_{1}, j_{2}}^{i, h_{2}}+\mathbf{e}_{i}\right\|_{L^{2}\left(\delta_{K, j_{1}, j_{2}}\right)} \leq C \sqrt{\left|\delta_{K, j_{1}, j_{2}}\right|},
\end{aligned}
$$

where the constant $C$ in (51) and (52) are independent off $H, h_{1}, h_{2}, \varepsilon_{1}, \varepsilon_{2}$.

The proof the this lemma follows the proves of Lemma 4.1 and Lemma 4.2, respectively for (52) and (51), by using the ellipticity of $a^{\varepsilon}=a(x, y, z)$ (see assumption (H1)).

As a preparation for the proof of the main theorem, we define $\widetilde{a}_{K}^{0}\left(x_{K, j_{1}}\right)$ as

$$
\left(\widetilde{a}_{K}^{0}\left(x_{K, j_{1}}\right)\right) \mathbf{e}_{i} \cdot \mathbf{e}_{k}=\frac{1}{\left|\delta_{K, j_{1}}\right|} \sum_{T_{K, j_{1}} \in \mathcal{T}_{K, j_{1}}} \sum_{j_{2}=1}^{J_{2}} \omega_{K, j_{1}, j_{2}} \bar{a}_{K, j_{1}}^{1}\left(x_{K, j_{1}, j_{2}}\right)\left(\nabla \widetilde{\chi}_{K, j_{1}}^{i, h_{1}}\left(x_{K, j_{1}, j_{2}}\right)+\mathbf{e}_{i}\right) \cdot \mathbf{e}_{k},(
$$


where $\tilde{\chi}_{K, j_{1}}^{i, h_{1}} \in S^{q_{1}}\left(\delta_{K, j_{1}}, \mathcal{T}_{K, j_{1}}\right)$ (or $\left.S_{0}^{q_{1}}\left(\delta_{K, j_{1}}, \mathcal{T}_{K, j_{1}}\right)\right)$ where $i=1, \cdots, d$ satisfies that for $\forall z^{h_{1}} \in S^{q_{1}}\left(\delta_{K, j_{1}}, \mathcal{T}_{K, j_{1}}\right)$ (or $S_{0}^{q_{1}}\left(\delta_{K, j_{1}}, \mathcal{T}_{K, j_{1}}\right)$ accordingly),

$$
\sum_{T_{K, j_{1}} \in \mathcal{T}_{K, j_{1}}} \sum_{j_{2}=1}^{J_{2}} \omega_{K, j_{1}, j_{2}} \bar{a}_{K, j_{1}}^{1}\left(x_{K, j_{1}, j_{2}}\right)\left(\nabla \widetilde{\chi}_{K, j_{1}}^{i, h_{1}}\left(x_{K, j_{1}, j_{2}}\right)+\mathbf{e}_{i}\right) \cdot \nabla z^{h_{1}}=0,
$$

where $\bar{a}_{K, j_{1}}^{1}\left(x_{K, j_{1}, j_{2}}\right)$ is defined in (34).

Proof of Theorem 5.5. We first split $r_{H M M}=\left\|a^{0}\left(x_{K, j_{1}}\right)-a^{0, h_{2}}\left(x_{K, j_{1}}\right)\right\|_{\mathcal{F}}$,

$$
\begin{aligned}
\left\|a^{0}\left(x_{K, j_{1}}\right)-a_{K}^{0}\left(x_{K, j_{1}}\right)\right\|_{\mathcal{F}} & \leq\left\|a^{0}\left(x_{K, j_{1}}\right)-\bar{a}_{K}^{0}\left(x_{K, j_{1}}\right)\right\|_{\mathcal{F}}+\left\|\bar{a}_{K}^{0}\left(x_{K, j_{1}}\right)-\widetilde{a}_{K}^{0}\left(x_{K, j_{1}}\right)\right\|_{\mathcal{F}} \\
& +\left\|\widetilde{a}_{K}^{0}\left(x_{K, j_{1}}\right)-a_{K}^{0}\left(x_{K, j_{1}}\right)\right\|_{\mathcal{F}} \\
& :=I_{1}+I_{2}+I_{3}
\end{aligned}
$$

where $a^{0}\left(x_{K, j_{1}}\right)$ is defined in (26), $a_{K}^{0}\left(x_{K, j_{1}}\right)$ is defined in (12) for $N=2, \bar{a}_{K}^{0}\left(x_{K, j_{1}}\right)$ is defined in (32) and $\widetilde{a}_{K}^{0}\left(x_{K, j_{1}}\right)$ is defined in (53).

Lemma 5.3 and Lemma 5.4 give respectively the following estimates for the term $I_{1}$ in (55), i.e.

$$
I_{1} \leq C\left(\frac{h_{1}}{\varepsilon_{1}}\right)^{2 q_{1}}
$$

for periodic boundary coupling or

$$
I_{1} \leq C\left(\frac{h_{1}}{\varepsilon_{1}}\right)^{2 q_{1}}+\frac{\varepsilon_{1}}{\delta_{1}}
$$

for Dirichlet boundary coupling.

For the term $I_{2}$, using (51) and Cauchy-Schwarz inequality we have

$$
\begin{aligned}
& \left|\left(\bar{a}_{K}^{0}\left(x_{K, j_{1}}\right)-\widetilde{a}_{K}^{0}\left(x_{K, j_{1}}\right)\right) \mathbf{e}_{i} \cdot \mathbf{e}_{k}\right| \\
& =\mid \frac{1}{\left|\delta_{K, j_{1}}\right|} \sum_{T_{K, j_{1}} \in \mathcal{T}_{K, j_{1}}} \sum_{j_{2}=1}^{J_{2}} \omega_{K, j_{1}, j_{2}}\left(\left(a^{1}\left(x_{K, j_{1}}, \frac{x_{K, j_{1}, j_{2}}}{\varepsilon_{1}}\right)-\bar{a}_{K, j_{1}}^{1}\left(x_{K, j_{1}, j_{2}}\right)\right)\left(\nabla \bar{\chi}_{K, j_{1}}^{i, h_{1}}\left(x_{K, j_{1}, j_{2}}\right)+\mathbf{e}_{i}\right) \cdot \mathbf{e}_{k}\right. \\
& \left.+\bar{a}_{K, j_{1}}^{1}\left(x_{K, j_{1}, j_{2}}\right)\left(\nabla \bar{\chi}_{K, j_{1}}^{i, h_{1}}-\nabla \widetilde{\chi}_{K, j_{1}}^{i, h_{1}}\right) \cdot \mathbf{e}_{k}\right) \mid \\
& \leq C\left(\max _{x_{K, j_{1}, j_{2}} \in \delta_{K, j_{1}}}\left\|a^{1}\left(x_{K, j_{1}}, \frac{x_{K, j_{1}, j_{2}}}{\varepsilon_{1}}\right)-\bar{a}_{K, j_{1}}^{1}\left(x_{K, j_{1}, j_{2}}\right)\right\|_{\mathcal{F}}+\frac{1}{\sqrt{\left|\delta_{K, j_{1}}\right|}}\left\|\nabla \bar{\chi}_{K, j_{1}}^{i, h_{1}}-\nabla \widetilde{\chi}_{K, j_{1}}^{i, h_{1}}\right\|_{L^{2}\left(\delta_{K, j_{1}}\right)}\right) .
\end{aligned}
$$

Using the ellipticity of $\bar{a}_{K, j_{1}}^{1}(x)$ (see Remark 5.2), we have

$$
\begin{aligned}
& \left.\lambda\left\|\nabla \bar{\chi}_{K, j_{1}}^{i, h_{1}}-\nabla \widetilde{\chi}_{K, j_{1}}^{i, h_{1}}\right\|_{L^{2}\left(\delta_{K, j_{1}}\right)}^{2}\right) \\
& \leq \sum_{T_{K, j_{1}} \in \mathcal{T}_{K, j_{1}}} \sum_{j_{2}=1}^{J_{2}} \omega_{K, j_{1}, j_{2}} \bar{a}_{K, j_{1}}^{1}\left(x_{K, j_{1}, j_{2}}\right)\left(\nabla \bar{\chi}_{K, j_{1}}^{i, h_{1}}-\nabla \widetilde{\chi}_{K, j_{1}}^{i, h_{1}}\right) \cdot \nabla\left(\bar{\chi}_{K, j_{1}}^{i, h_{1}}-\nabla \widetilde{\chi}_{K, j_{1}}^{i, h_{1}}\right) \\
& =\sum_{T_{K, j_{1}} \in \mathcal{T}_{K, j_{1}}} \sum_{j_{2}=1}^{J_{2}} \omega_{K, j_{1}, j_{2}}\left(\bar{a}_{K, j_{1}}^{1}\left(x_{K, j_{1}, j_{2}}\right)-a^{1}\left(x_{K, j_{1}}, \frac{x_{K, j_{1}, j_{2}}}{\varepsilon_{1}}\right)\right)\left(\nabla \bar{\chi}_{K, j_{1}}^{i, h_{1}}+\mathbf{e}_{i}\right) \cdot\left(\nabla \bar{\chi}_{K, j_{1}}^{i, h_{1}}-\nabla \widetilde{\chi}_{K, j_{1}}^{i, h_{1}}\right) \\
& \leq C \sqrt{\left|\delta_{K, j_{1}}\right|} \max _{x_{K, j_{1}, j_{2}} \in \delta_{K, j_{1}}}\left\|a^{1}\left(x_{K, j_{1}}, \frac{x_{K, j_{1}, j_{2}}}{\varepsilon_{1}}\right)-\bar{a}_{K, j_{1}}^{1}\left(x_{K, j_{1}, j_{2}}\right)\right\|_{\mathcal{F}}\left\|\nabla \bar{\chi}_{K, j_{1}}^{i, h_{1}}-\nabla \widetilde{\chi}_{K, j_{1}}^{i, h_{1}}\right\|_{L^{2}\left(\delta_{K, j_{1}}\right)} .
\end{aligned}
$$


The last inequality above is obtained by using Cauchy-Schwarz inequality and (51). Hence, we obtain

$$
\frac{1}{\sqrt{\left|\delta_{K, j_{1} \mid}\right|}}\left\|\nabla \bar{\chi}_{K, j_{1}}^{i, h_{1}}-\nabla \widetilde{\chi}_{K, j_{1}}^{i, h_{1}}\right\|_{L^{2}\left(\delta_{K, j_{1}}\right)} \leq C \max _{x_{K, j_{1}, j_{2}} \in \delta_{K, j_{1}}}\left\|a^{1}\left(x_{K, j_{1}}, \frac{x_{K, j_{1}, j_{2}}}{\varepsilon_{1}}\right)-\bar{a}_{K, j_{1}}^{1}\left(x_{K, j_{1}, j_{2}}\right)\right\|_{\mathcal{F}} .
$$

In view of (56) and (58), the term $I_{2}$ has the upper bound

$$
I_{2} \leq C\left(\left(\frac{h_{2}}{\varepsilon_{2}}\right)^{2 q_{2}}+\left(\frac{h_{1}}{\varepsilon_{1}}\right)^{2 q_{1}}\right)
$$

for periodic boundary coupling (using Lemma 5.3), or

$$
I_{2} \leq C\left(\left(\frac{h_{2}}{\varepsilon_{2}}\right)^{2 q_{2}}+\left(\frac{h_{1}}{\varepsilon_{1}}\right)^{2 q_{1}}+\frac{\varepsilon_{2}}{\delta_{2}}\right)
$$

for Dirichlet boundary coupling (using Lemma 5.4).

For the term $I_{3}$ of (55), similarly to (56), we first have

$$
\begin{aligned}
\left|\left(\widetilde{a}_{K}^{0}\left(x_{K, j_{1}}\right)-a_{K}^{0}\left(x_{K, j_{1}}\right)\right) \mathbf{e}_{i} \cdot \mathbf{e}_{k}\right| \leq C\left(\max _{x_{K, j_{1}, j_{2}} \in \delta_{K, j_{1}}}\left\|\bar{a}_{K, j_{1}}^{1}\left(x_{K, j_{1}, j_{2}}\right)-a_{K, j_{1}}^{1}\left(x_{K, j_{1}, j_{2}}\right)\right\|_{\mathcal{F}}\right. \\
\left.+\frac{1}{\sqrt{\left|\delta_{K, j_{1}}\right|}}\left\|\nabla \widetilde{\chi}_{K_{j}}^{i, h_{1}}-\nabla \chi_{K_{j}}^{i, h_{1}}\right\|_{L^{2}\left(\delta_{K, j_{1}}\right)}\right) .
\end{aligned}
$$

Similarly to (57), we obtain

$$
\begin{aligned}
& \lambda\left\|\nabla \chi_{K_{j}}^{i, h_{1}}-\nabla \widetilde{\chi}_{K_{j}}^{i, h_{1}}\right\|_{L^{2}\left(\delta_{K, j_{1}}\right)}^{2} \\
& \leq C \max _{x_{K, j_{1}, j_{2}} \in \delta_{K, j_{1}}}\left\|\bar{a}_{K, j_{1}}^{1}\left(x_{K, j_{1}, j_{2}}\right)-a_{K, j_{1}}^{1}\left(x_{K, j_{1}, j_{2}}\right)\right\|_{\mathcal{F}}\left\|\nabla \widetilde{\chi}_{K, j_{1}}^{i, h_{1}}+\mathbf{e}_{i}\right\|_{L^{2}\left(\delta_{K, j_{1}}\right)}\left\|\nabla \widetilde{\chi}_{K, j_{1}}^{i, h_{1}}-\nabla \chi_{K, j_{1}}^{i, h_{1}}\right\|_{L^{2}\left(\delta_{K, j_{1}}\right)} .
\end{aligned}
$$

In view of $\left\|\nabla \widetilde{\chi}_{K, j_{1}}^{i, h_{1}}+\mathbf{e}_{i}\right\|_{L^{2}\left(\delta_{K, j_{1}}\right)} \leq C \sqrt{\left|\delta_{K, j_{1}}\right|}$, (which can be obtained following the proof of Lemma 4.2), the following inequality holds,

$$
\frac{1}{\sqrt{\left|\delta_{K, j_{1}}\right|}}\left\|\nabla \chi_{K, j_{1}}^{i, h_{1}}-\nabla \widetilde{\chi}_{K, j_{1}}^{i, h_{1}}\right\|_{L^{2}\left(\delta_{K, j_{1}}\right)} \leq C \max _{x_{K, j_{1}, j_{2}} \in \delta_{K, j_{1}}}\left\|\bar{a}_{K, j_{1}}^{1}\left(x_{K, j_{1}, j_{2}}\right)-a_{K, j_{1}}^{1}\left(x_{K, j_{1}, j_{2}}\right)\right\|_{\mathcal{F}} .
$$

The last step is to estimate $\left\|\bar{a}_{K, j_{1}}^{1}\left(x_{K, j_{1}, j_{2}}\right)-a_{K, j_{1}}^{1}\left(x_{K, j_{1}, j_{2}}\right)\right\|_{\mathcal{F}}$. By simple computation, we derive

$$
\begin{aligned}
& \left|\left(\bar{a}_{K, j_{1}}^{1}\left(x_{K, j_{1}, j_{2}}\right)-a_{K, j_{1}}^{1}\left(x_{K, j_{1}, j_{2}}\right)\right) \mathbf{e}_{i} \cdot \mathbf{e}_{k}\right| \\
& =\frac{1}{\left|\delta_{K, j_{1}, j_{2}}\right|} \mid \int_{\delta_{K, j_{1}, j_{2}}}\left(a\left(x, \frac{x}{\varepsilon_{1}}, \frac{x}{\varepsilon_{2}}\right)\left(\nabla \chi_{K, j_{1}, j_{2}}^{i, h_{2}}-\nabla \bar{\chi}_{K, j_{1}, j_{2}}^{i, h_{2}}\right) \cdot\left(\nabla \bar{\chi}_{K, j_{1}, j_{2}}^{k, h_{2}}+\mathbf{e}_{k}\right)\right. \\
& \left.+\left(a\left(x_{K, j_{1}}, \frac{x_{K, j_{1}, j_{2}}}{\varepsilon_{1}}, \frac{x}{\varepsilon_{2}}\right)-a\left(x, \frac{x}{\varepsilon_{1}}, \frac{x}{\varepsilon_{2}}\right)\right)\left(\nabla \bar{\chi}_{K, j_{1}, j_{2}}^{i, h_{2}}+\mathbf{e}_{i}\right) \cdot\left(\nabla \bar{\chi}_{K, j_{1}, j_{2}}^{k, h_{2}}+\mathbf{e}_{k}\right)\right) d x \mid .
\end{aligned}
$$

Applying (52) to (63), we then obtain

$$
\begin{aligned}
\left|\left(\bar{a}_{K, j_{1}}^{1}\left(x_{K, j_{1}, j_{2}}\right)-a_{K, j_{1}}^{1}\left(x_{K, j_{1}, j_{2}}\right)\right) \mathbf{e}_{i} \cdot \mathbf{e}_{k}\right| & \leq C\left(\max _{x \in \delta_{K, j_{1}, j_{2}}}\left\|a\left(x_{K, j_{1}}, \frac{x_{K, j_{1}, j_{2}}}{\varepsilon_{1}}, \frac{x}{\varepsilon_{2}}\right)-a\left(x, \frac{x}{\varepsilon_{1}}, \frac{x}{\varepsilon_{2}}\right)\right\|_{\mathcal{F}}\right. \\
& \left.+\frac{1}{\sqrt{\left|\delta_{K, j_{1}, j_{2}}\right|}}\left\|\nabla \chi_{K, j_{1}, j_{2}}^{i, h_{2}}-\nabla \bar{\chi}_{K, j_{1}, j_{2}}^{i, h_{2}}\right\|_{L^{2}\left(\delta_{K, j_{1}}\right)}\right) .
\end{aligned}
$$


The following inequality can be obtained by the ellipticity assumption of $a^{\varepsilon}(x)$,

$$
\frac{\lambda}{\sqrt{\left|\delta_{K, j_{1} \mid}\right|}}\left\|\nabla \chi_{K, j_{1}, j_{2}}^{i, h_{2}}-\nabla \bar{\chi}_{K, j_{1}, j_{2}}^{i, h_{2}}\right\|_{L^{2}\left(\delta_{K, j_{1}}\right)} \leq C \max _{x \in \delta_{K, j_{1}, j_{2}}}\left\|a\left(x_{K, j_{1}}, \frac{x_{K, j_{1}, j_{2}}}{\varepsilon_{1}}, \frac{x}{\varepsilon_{2}}\right)-a\left(x, \frac{x}{\varepsilon_{1}}, \frac{x}{\varepsilon_{2}}\right)\right\|_{\mathcal{F}}(
$$

Since we assume $a(x, y, z)$ Lipschitz continuous on $\bar{\Omega} \times \bar{Y} \times \bar{Z}$, then

$$
\max _{x \in \delta_{K, j_{1}, j_{2}}}\left\|a\left(x_{K, j_{1}}, \frac{x_{K, j_{1}, j_{2}}}{\varepsilon_{1}}, \frac{x}{\varepsilon_{2}}\right)-a\left(x, \frac{x}{\varepsilon_{1}}, \frac{x}{\varepsilon_{2}}\right)\right\|_{\mathcal{F}} \leq C\left(\delta_{1}+\frac{\delta_{2}}{\varepsilon_{1}}\right) .
$$

Therefore, the term $I_{3}$ can be bounded by

$$
I_{3}=\left\|\widetilde{a}_{K}^{0}\left(x_{K, j_{1}}\right)-a_{K}^{0}\left(x_{K, j_{1}}\right)\right\|_{\mathcal{F}} \leq C\left(\delta_{1}+\frac{\delta_{2}}{\varepsilon_{1}}\right) .
$$

Furthermore if $a\left(x, \frac{x}{\varepsilon_{1}}, \frac{x}{\varepsilon_{2}}\right)$ is collocated on the quadrature points, i.e. $a\left(x, \frac{x_{K, j_{1}}}{\varepsilon_{1}}, \frac{x_{K, j_{1}, j_{2}}}{\varepsilon_{2}}\right)$, then $I_{3}=0$.

Generalization to $N+1$ scale problems. Following the idea of the proof for Theorem 5.5, one can generalize the result to the $N+1$ scale FE-HMM. Assuming $a^{\varepsilon}=a\left(x, \frac{x}{\varepsilon_{1}}, \cdots, \frac{x}{\varepsilon_{N}}\right)$ and similar assumptions as stated in Theorem 5.5 for an $N+1$ scale tensor, then the following error estimate holds

$$
r_{H M M} \leq C\left(\left(\frac{h_{1}}{\varepsilon_{1}}\right)^{2 q_{1}}+\cdots+\left(\frac{h_{N}}{\varepsilon_{N}}\right)^{2 q_{N}}+r_{M O D}\right) .
$$

- If the cell problems at each scale are coupled with periodic boundary conditions and $\delta_{s} / \varepsilon_{s} \in \mathbb{N}, s=1, \cdots, N$, then we have

$$
r_{M O D} \leq C\left(\delta_{1}+\frac{\delta_{2}}{\varepsilon_{1}}+\cdots+\frac{\delta_{N}}{\varepsilon_{N-1}}\right) .
$$

In addition, if all the cell problems are collocated at the corresponding quadrature points, then

$$
r_{M O D}=0
$$

- If the cell problem at each scale are coupled with Dirichlet boundary conditions with $\delta_{s}>\varepsilon_{s}, s=1, \cdots, N$, then

$$
r_{M O D} \leq C\left(\delta_{1}+\frac{\delta_{2}}{\varepsilon_{1}}+\cdots+\frac{\delta_{N}}{\varepsilon_{N-1}}+\frac{\varepsilon_{1}}{\delta_{1}}+\cdots+\frac{\varepsilon_{N}}{\delta_{N}}\right) .
$$

In addition, if all the cell problems are collocated at the corresponding quadrature points, then

$$
r_{M O D} \leq C\left(\frac{\varepsilon_{1}}{\delta_{1}}+\cdots+\frac{\varepsilon_{N}}{\delta_{N}}\right)
$$


Complexity. Let $M_{0}$ be the the number of DOF of the macro FEM. We write $\hat{h}_{s}=\frac{h_{s}}{\varepsilon_{s}}$ and denote $M_{s}=\mathcal{O}\left(\hat{h}_{s}^{-d}\right)$ the number of degrees of freedom (DOF) for cell problems on the scale $s$, where $s=1, \cdots, N$. We emphasize that $M_{s}$ is independent of $\varepsilon_{s}$ since $\left|\delta_{K, j_{1}, \cdots, j_{s}}\right|=\delta_{s}^{d}=$ $\left(\frac{\delta_{s}}{\varepsilon_{s}}\right)^{d} \varepsilon_{s}^{d}=C_{s} \varepsilon_{s}^{d}$ with $C_{s}$ a moderate constant (recall our assumption that $\delta_{s}=\hat{C}_{s} \varepsilon_{s}$ ). Using quasi-uniform meshes for each scale, we have the following relations

$$
H=\mathcal{O}\left(M_{0}^{-1 / d}\right), \quad \hat{h}_{s}=\mathcal{O}\left(M_{s}^{-1 / d}\right), s=1, \cdots, N .
$$

In view of (23), (24) and (67), optimal convergence rates (up to a modeling error $r_{M O D}$ independent of $H, h_{s}$, where $\left.s=1, \cdots, N\right)$ can be obtained for quasi-uniform meshes given by,

$$
\hat{h}_{s} \approx H^{\frac{\ell}{2 q_{s}}} \text { for the } H^{1} \text { norm, } \hat{h}_{s} \approx H^{\frac{\ell+1}{2 q_{s}}} \text { for the } L^{2} \text { norm. }
$$

The corresponding complexity in term of macro DOF reads

$$
\begin{aligned}
& \mathcal{O}(\underbrace{H^{-d}}_{M_{0}} \cdot \underbrace{H^{\frac{-d \ell}{2 q_{1}}}}_{M_{1}} \cdots \cdots \cdot \underbrace{H^{\frac{-d \ell}{2 q_{N}}}}_{M_{N}})=\mathcal{O}\left(M_{0}^{1+\frac{\ell}{2 q_{1}}+\cdots+\frac{\ell}{2 q_{N}}}\right) \text { for the } H^{1} \text { norm, } \\
& \mathcal{O}(\underbrace{H^{-d}}_{M_{0}} \cdot \underbrace{H^{\frac{-d(\ell+1)}{2 q_{1}}}}_{M_{1}} \cdots \cdots \underbrace{H^{\frac{-d(\ell+1)}{2 q_{N}}}}_{M_{N}})=\mathcal{O}\left(M_{0}^{1+\frac{(\ell+1)}{2 q_{1}}+\cdots+\frac{(\ell+1)}{2 q_{N}}}\right) \text { for the } L^{2} \text { norm. }
\end{aligned}
$$

\section{Numerical Experiment}

We consider problem (1) on the domain $\Omega=[0,1]^{2}$ with $f=1$ and the multiscale tensor

$$
a^{\varepsilon}(x)=\left(x_{1}^{2}+x_{2}^{2}+1\right)\left(\begin{array}{cc}
\sin \left(2 \pi \frac{x_{1}}{\varepsilon_{1}}+2\right) \cos \left(2 \pi \frac{x_{1}}{\varepsilon_{2}}+2\right) & 0 \\
0 & \sin \left(2 \pi \frac{x_{2}}{\varepsilon_{1}}+2\right) \cos \left(2 \pi \frac{x_{2}}{\varepsilon_{2}}+2\right)
\end{array}\right) .
$$

where $\varepsilon_{1}=5 \times 10^{-3}, \varepsilon_{2}=5 \times 10^{-5}$. The corresponding homogenized tensor is

$$
a^{0}(x)=3\left(x_{1}^{2}+x_{2}^{2}+1\right) I_{2} .
$$

In this experiment, we use FEM with piecewise linear basis functions on triangle elements (called P1-FEM) as the solver for problems on all three scales. For the QFs, we choose the barycenter of each element as the quadrature node for both macro and meso scales which satisfies the assumptions on the QFs stated in both Theorem 4.4 and 5.5 for $P 1$ triangle elements. We take the P1-FE solution $u^{0, H}$ of (3) as the reference solution which is computed on a $1024 \times 1024$ uniform triangular mesh. We use uniform triangulation in the FE-HMM procedure and denote $N_{m a c}, N_{m e s}, N_{m i c}$ as the degrees of freedom (DOF) of one direction in the macro, meso and micro partitions respectively, i.e. $H=1 / N_{m a c}, h_{1} / \varepsilon_{1}=1 / N_{\text {mes }}$, $h_{2} / \varepsilon_{2}=N_{m i c}$. In Fig. 2, we observe that the $H^{1}$ error decays with a rate of $\mathcal{O}(H)$ and the $L^{2}$ error decays as $\mathcal{O}\left(H^{2}\right)$, which confirms the results in Theorem 4.4 and 5.5.

In Fig. 3, we show the error behavior with $N_{\text {mes }}$ is fixed at $2,4,8,16$ while macro and micro meshes are refined simultaneously. We conclude that the theoretical convergence rate can only be obtained when the meshes are refined simultaneously and that our error estimates (at least for three scale problems) are sharp.

Acknowledgements. This work was supported in part by the Swiss National Foundation Grant 200021 134716/1. 


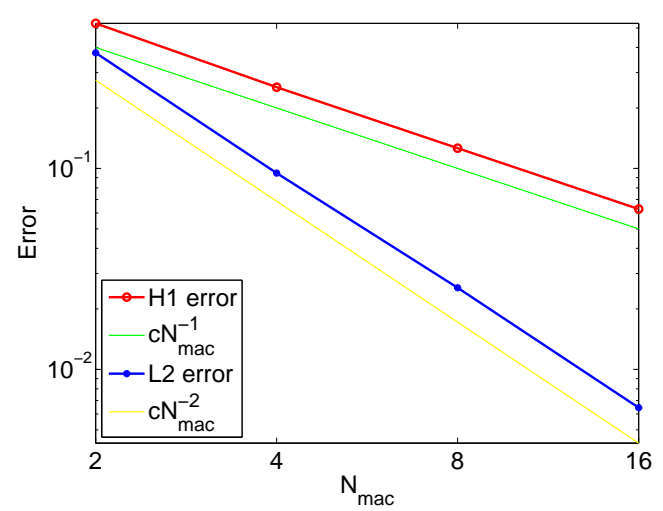

Figure 2: We set $N_{m e s}=N_{m i c}=N_{m a c}^{1 / 2}$ for computing $H^{1}$ error and $N_{m e s}=N_{m i c}=N_{m a c}$ for $L^{2}$ error.

\section{References}

[1] A. Abdulle. On a priori error analysis of fully discrete heterogeneous multiscale FEM. SIAM, Multiscale Model. Simul., 4(2):447-459, 2005.

[2] A. Abdulle. Analysis of a heterogeneous multiscale FEM for problems in elasticity. Math. Models Methods Appl. Sci., 16(4):615-635, 2006.

[3] A. Abdulle. The finite element heterogeneous multiscale method: a computational strategy for multiscale pdes. GAKUTO Int. Ser. Math. Sci. Appl., 31:135-184, 2009.

[4] A. Abdulle. A priori and a posteriori error analysis for numerical homogenization: a unified framework. Ser. Contemp. Appl. Math. CAM, 16:280-305, 2011.

[5] A. Abdulle and Y. Bai. Reduced basis finite element heterogeneous multiscale method for high-order discretizations of elliptic homogenization problems. J. Comput. Phys., 231(21):7014-7036, 2012.

[6] A. Abdulle, W. E, B. Engquist, and E. Vanden-Eijnden. The heterogeneous multiscale method. Acta Numer., 21:1-87, 2012.

[7] A. Abdulle and G. Vilmart. Analysis of the finite element heterogeneous multiscale method for quasilinear elliptic homogenization problems. To appear in Math. Comp., 2012 .

[8] G. Allaire. Homogenization and two-scale convergence. SIAM J. Math. Anal., 23(6):14821518, 1992.

[9] G. Allaire and M. Briane. Multiscale convergence and reiterated homogenisation. Proc. Roy. Soc. Edinburgh Sect. A, 126(2):297-342, 1996.

[10] A. Bensoussan, J.-L. Lions, and G. Papanicolaou. Asymptotic analysis for periodic structures. North-Holland Publishing Co., Amsterdam, 1978.

[11] P. Ciarlet. Basic error estimates for Elliptic problems, volume 2 of Handbook of numerical analysis. Elsevier science publishers B.V., North-Holland, 1991. 


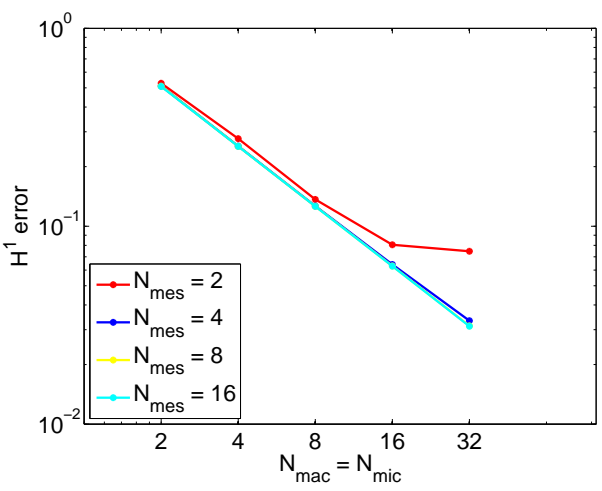

(a) $H^{1}$ Error with fixed $N_{m e s}$

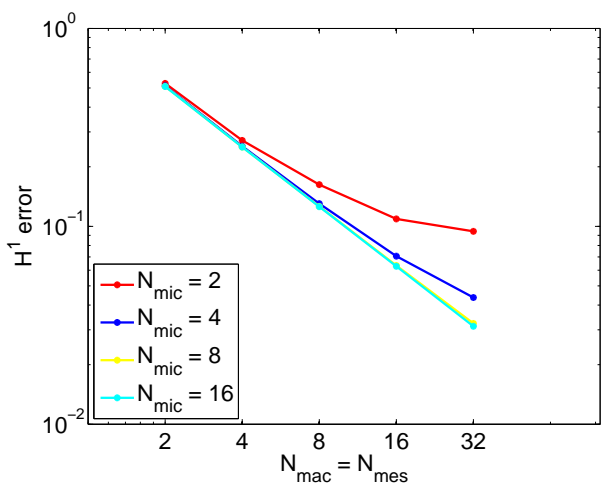

(c) $H^{1}$ Error with fixed $N_{m i c}$

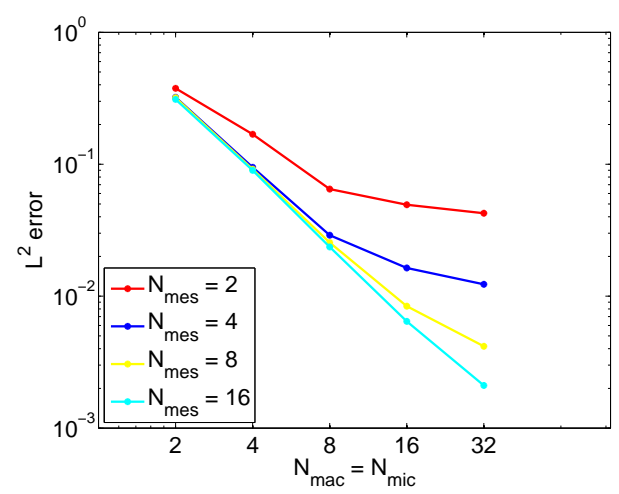

(b) $L^{2}$ Error with fixed $N_{m e s}$

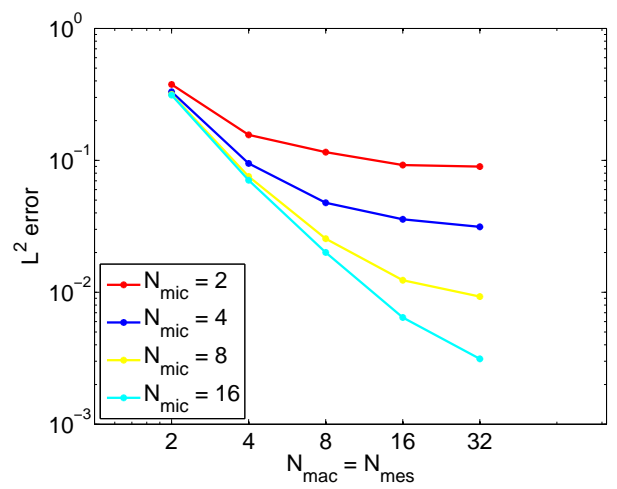

(d) $L^{2}$ Error with fixed $N_{m i c}$

Figure 3: The convergence behavior when $N_{m i c}$ or $N_{\text {mes }}$ is fixed at different values. 
[12] P. Ciarlet and P. Raviart. The combined effect of curved boundaries and numerical integration in isoparametric finite element methods. Math. Foundation of the FEM with Applications to PDE, pages 409-474, 1972.

[13] E. De Giorgi and S. Spagnolo. Sulla convergenza degli integrali dell'energia per operatori ellittici del secondo ordine. Boll. Un. Mat. Ital., 4(8):391-411, 1973.

[14] R. Du and P. Ming. Heterogeneous multiscale finite element method with novel numerical integration schemes. Commm. Math. Sci., 8(4):863-885, 2010.

[15] W. E. Principles of Multiscale Modeling. Cambridge university press, New York, 2011.

[16] W. E and B. Engquist. The heterogeneous multiscale methods. Commun. Math. Sci., $1(1): 87-132,2003$.

[17] W. E, P. Ming, and P. Zhang. Analysis of the heterogeneous multiscale method for elliptic homogenization problems. J. Amer. Math. Soc., 18(1):121-156, 2005.

[18] Y. Efendiev and T. Y. Hou. Multiscale finite element methods. Theory and applications, volume 4 of Surveys and Tutorials in the Applied Mathematical Sciences. Springer, New York, 2009.

[19] Y. Efendiev and X. Wu. Multiscale finite element for problems with highly oscillatory coefficients. Numer. Math., 90:459-486, 2002.

[20] J. Fish. Multiscale methods: bridging the scales in science and engineering. Oxford University Press, New York, 2009.

[21] V. H. Hoang and C. Schwab. High-dimensional finite elements for elliptic problems with multiple scales. Multiscale Model. Simul, 3(1):168-194, 2005.

[22] V. Jikov, S. Kozlov, and O. Oleinik. Homogenization of differential operators and integral functionals. Springer-Verlag, Berlin, Heidelberg, 1994.

[23] F. Murat and L. Tartar. H-convergence, topics in the mathematical modeling of composite materials. Progr. Nonlinear Differential Equations Appl., 31:21-43, 1997.

[24] G. Nguetseng. A general convergence result for a functional related to the theory of homogenization. SIAM J. Math. Anal., 20(3):608-623, 1989.

[25] E. Sanchez-Palencia. Nonhomogeneous Media and Vibration Theory. Lecture Notes in Phys. 127. Springer, Berlin, 1980.

[26] L. Tartar. Incompressible fluid flow through porous media convergence of the homogenization process. in appendix of [25]. Springer, Berlin, 1980. 


\section{Appendix}

\section{Numerical integration error analysis for simplicial elements}

In this section, we analyse the numerical integration error which we use in Section 4 for the estimation of (58).

Let $E_{T}(\phi)$ denote the numerical integration error on the element $T$ defined as

$$
E_{T}(\phi)=\int_{T} \phi d x-\sum_{j=1}^{J} \omega_{j} \phi\left(x_{K, j_{1}}\right)
$$

and $E_{\hat{T}}(\hat{\phi})$ is the corresponding error on the reference element $\hat{T}$

$$
E_{\hat{T}}(\hat{\phi})=\int_{\hat{T}} \hat{\phi} d \hat{x}-\sum_{j=1}^{J} \hat{\omega}_{j} \phi\left(\hat{x}_{K, j_{1}}\right) .
$$

Theorem .1. (Global higher order numerical integration error theorem) Assume that $a_{i j} \in$ $W^{2 m, \infty}(\mathcal{D})$ and $u, w \in W(\mathcal{D}) \cap H^{m+1}(\mathcal{D})(W(\mathcal{D})$ is defined as in $(7)$ if $\mathcal{D}$ is rectangular or otherwise as in (8)) are the solutions of the following problems: for $\forall v \in W(\mathcal{D})$,

$$
\begin{aligned}
& \int_{\mathcal{D}} a \nabla u \cdot \nabla v d x=\int_{\mathcal{D}} f_{1} v d x, \\
& \int_{\mathcal{D}} a \nabla w \cdot \nabla v d x=\int_{\mathcal{D}} f_{2} v d x
\end{aligned}
$$

and $u^{h}, w^{h}$ are the FEM solutions with numerical quadrature in $S^{m}\left(\mathcal{D}, \mathcal{T}_{h}\right)$ where $\mathcal{T}_{h}$ is a shape regular partition of $\mathcal{D}$ and elements $T \in \mathcal{T}_{h}$ are quasi-uniform. Assume the numerical quadrature satisfies

$$
E_{\hat{T}}(\hat{\phi})=0, \quad \forall \hat{\phi} \in \mathcal{P}^{2 m-1}(\hat{T})
$$

Then we have

$$
\begin{aligned}
& \left|\int_{\mathcal{D}} \sum_{i j} a_{i j} \partial_{i} u^{h} \partial_{j} w^{h} d x-\sum_{T \in \mathcal{T}_{h}} \sum_{j=1}^{J} \omega_{T_{j}} \sum_{i j}\left(a_{i j} \partial_{i} u^{h} \partial_{j} w^{h}\right)\left(x_{T_{j}}\right)\right| \\
& \leq C h^{2 m}\left(\sum_{i j}\left\|a_{i j}\right\|_{W^{2 m, \infty}(\mathcal{D})}\right)\|u\|_{H^{m}(\mathcal{D})}\|w\|_{H^{m}(\mathcal{D})} .
\end{aligned}
$$

Proof. We first write

$$
E_{T}\left(a_{i j} \partial_{i} u^{h} \partial_{j} w^{h}\right)=\int_{T} \sum_{i j} a_{i j} \partial_{i} u^{h} \partial_{j} w^{h} d x-\sum_{j}^{J} \omega_{K, j_{1}, j_{2}} \sum_{i j}\left(a_{i j} \partial_{i} u^{h} \partial_{j} w^{h}\right)\left(x_{T_{j}}\right) .
$$

Using Bramble-Hilbert lemma (see [11]), we can obtain the following local estimate

$$
\left|E_{T}\left(a_{i j} \partial_{i} u^{h} \partial_{j} w^{h}\right)\right| \leq C h^{2 m}\left(\sum_{i j}\left\|a_{i j}\right\|_{W^{2 m, \infty}(T)}\right)\left\|u^{h}\right\|_{H^{m}(T)}\left\|w^{h}\right\|_{H^{m}(T)} .
$$


Thus, we obtain

$$
\begin{aligned}
& \left|\int_{\mathcal{D}} \sum_{i j} a_{i j} \partial_{i} u^{h} \partial_{j} w^{h} d x-\sum_{T_{K, j_{1}} \in \mathcal{T}_{K, j_{1}}} \sum_{j_{2}=1}^{J_{2}} \omega_{K, j_{1}, j_{2}} \sum_{i j}\left(a_{i j} \partial_{i} u^{h} \partial_{j} w^{h}\right)\left(x_{T_{j}}\right)\right| \\
& \leq C h^{2 m}\left(\sum_{i j}\left\|a_{i j}\right\|_{W^{2 m, \infty}(\mathcal{D})}\right) \sum_{T \in \mathcal{T}_{h}}\left\|u^{h}\right\|_{H^{m}(T)}\left\|w^{h}\right\|_{H^{m}(T)} \\
& =C h^{2 m}\left(\sum_{i j}\left\|a_{i j}\right\|_{W^{2 m, \infty}(\mathcal{D})}\right) \sum_{T \in \mathcal{T}_{h}}\left(\left(\sum_{\ell=1}^{m}\left|u^{h}\right|_{H^{\ell}(T)}^{2}\right)^{1 / 2}\left(\sum_{\ell=1}^{m}\left|w^{h}\right|_{H^{\ell}(T)}^{2}\right)^{1 / 2}\right) \\
& \leq C h^{2 m}\left(\sum_{i j}\left\|a_{i j}\right\|_{W^{2 m, \infty}(\mathcal{D})}\right)\left(\sum_{T \in \mathcal{T}_{h}} \sum_{\ell=1}^{m}\left|u^{h}\right|_{H^{\ell}(T)}^{2}\right)^{1 / 2}\left(\sum_{T \in \mathcal{T}_{h}} \sum_{\ell=1}^{m}\left|w^{h}\right|_{H^{\ell}(T)}^{2}\right)^{1 / 2} .
\end{aligned}
$$

Now we define $\Pi_{T}^{u} \in \mathcal{P}^{m}(T)$ which is an interpolation polynomial of $u$ on element $T$ (respectively $\Pi_{T}^{w}$ the interpolation polynomial of $w$ ). By classical interpolation error estimate (see [11, Chapter 3]), we have

$$
\left\|u-\Pi_{T}^{u}\right\|_{H^{\ell}(T)} \leq C h^{m+1-\ell}|u|_{H^{m+1}(T)}, \text { for } \ell \leq m .
$$

Furthermore, we can derive that for $\ell \leq m$

$$
\left\|\Pi_{T}^{u}\right\|_{H^{\ell}(T)} \leq\left\|u-\Pi_{T}^{u}\right\|_{H^{\ell}(T)}+\|u\|_{H^{\ell}(T)} \leq C\|u\|_{H^{m}(T)} .
$$

Using inverse inequality (assume that the triangulation $\mathcal{T}_{h}$ is quasi-uniform), we have

$$
\begin{aligned}
\sum_{\ell=1}^{m}\left|u^{h}\right|_{H^{\ell}(T)}^{2} & \leq \sum_{\ell=1}^{m}\left(\left|u^{h}-\Pi_{T}^{u}\right|_{H^{\ell}(T)}^{2}+\left|\Pi_{T}^{u}\right|_{H^{\ell}(T)}^{2}\right) \\
& \leq\left\|\Pi_{T}^{u}\right\|_{H^{m}(T)}^{2}+C \sum_{\ell=1}^{m} h^{-2 \ell+2}\left|u^{h}-\Pi_{T}^{u}\right|_{H^{1}(T)}^{2} \\
& \leq C\left(\|u\|_{H^{m}(T)}^{2}+\sum_{\ell=1}^{m} h^{-2 \ell+2}\left(\left|u^{h}-u\right|_{H^{1}(T)}^{2}+\left|u-\Pi_{T}^{u}\right|_{H^{1}(T)}^{2}\right)\right) \\
& \leq C\left(\|u\|_{H^{m}(T)}^{2}+h^{-2 m+2}\left\|u^{h}-u\right\|_{H^{1}(T)}^{2}\right) .
\end{aligned}
$$

Sum up the above inequality with respect to element $T \in \mathcal{T}_{h}$ and we obtain

$$
\sum_{T \in \mathcal{T}_{h}} \sum_{\ell=1}^{m}\left|u^{h}\right|_{H^{\ell}(T)}^{2} \leq C\left(\|u\|_{H^{m}(\mathcal{D})}^{2}+h^{-2 m+2}\left\|u-u^{h}\right\|_{H^{1}(\mathcal{D})}^{2}\right) .
$$

Using standard FEM error analysis with numerical quadrature (see for example [11]), we have the following result

$$
\left\|u-u^{h}\right\|_{H^{1}(\mathcal{D})} \leq C h^{m-1}|u|_{H^{m}(\mathcal{D})} .
$$

Combining (72) with (73), we have

$$
\sum_{T \in \mathcal{T}_{h}} \sum_{\ell=1}^{m}\left|u^{h}\right|_{H^{\ell}(T)}^{2} \leq C\|u\|_{H^{m}(\mathcal{D})} .
$$

Taking (74) into (71), we have proved the stated result. 


\section{Numerical integration error analysis for parallelogram elements}

In order to obtain the same error result for parallelogram elements, one needs to introduce the following semi-norm for space $W^{m, p}(\mathcal{D})$

$$
\{v\}_{W^{m, p}(\mathcal{D})}=\left(\int_{\mathcal{D}} \sum_{i=1}^{d}\left(\partial_{i}^{m} v\right)^{p}\right)^{1 / p},
$$

where $\partial_{i}:=(\partial) /\left(\partial x_{i}\right)$ (see for example [11], where such norms have been discussed). Based on this semi-norm, a corresponding Bramble-Hilbert lemma can be derived for parallelogram elements (see [11, Chapter 11]).

Theorem .2. (Bramble-Hilbert lemma for parallelogram elements.) Let $\mathcal{D}$ be a domain in $\mathbb{R}^{d}$, let $k \geq 0$ be an integer, let $p$ be a number satisfying $1 \leq p \leq \infty$, and let $\mathcal{L}$ be a continuous linear functional on the space $W^{m, p}(\mathcal{D})$ with the property that

$$
\mathcal{L}(q)=0 \text { for all } q \in \mathcal{Q}^{m-1}(\mathcal{D}) .
$$

Then there exists a constant $C$ which depends on $\mathcal{D}$ such that

$$
|\mathcal{L}(v)| \leq C\|\mathcal{L}\|_{W^{m, p}(\mathcal{D})}^{*}\{v\}_{W^{m, p}(\mathcal{D})}, \quad \forall v \in W^{m, p}(\mathcal{D}),
$$

where $\|\cdot\|_{W^{m, p}(\mathcal{D})}^{*}$ is the dual norm of space $\left.W^{m, p}(\mathcal{D})\right)$.

With the help of Theorem .2, a numerical integration error estimate can be obtained following the proof of Theorem .1.

Theorem .3. (Global higher order numerical integration error theorem for parallelogram elements) Assume that $a_{i j} \in W^{2 m, \infty}(\mathcal{D})$ and $u, w \in W(\mathcal{D}) \cap H^{m+1}(\mathcal{D})(W(\mathcal{D})$ is defined as in (7) if $\mathcal{D}$ is rectangular or otherwise as in (8)) are the solutions of the following problems: for $\forall v \in W(\mathcal{D})$,

$$
\begin{aligned}
& \int_{\mathcal{D}} a \nabla u \cdot \nabla v d x=\int_{\mathcal{D}} f_{1} v d x, \\
& \int_{\mathcal{D}} a \nabla w \cdot \nabla v d x=\int_{\mathcal{D}} f_{2} v d x
\end{aligned}
$$

and $u^{h}, w^{h}$ are the FEM solutions with numerical quadrature in $S^{m}\left(\mathcal{D}, \mathcal{T}_{h}\right)$ where $\mathcal{T}_{h}$ is a shape regular partition of $\mathcal{D}$ and elements $T \in \mathcal{T}_{h}$ are parallelogram and quasi-uniform. Assume the numerical quadrature satisfies

$$
E_{\hat{T}}(\hat{\phi})=0, \quad \forall \hat{\phi} \in \mathcal{Q}^{2 m-1}(\hat{T}) .
$$

Then we have

$$
\begin{aligned}
& \left|\int_{\mathcal{D}} \sum_{i j} a_{i j} \partial_{i} u^{h} \partial_{j} w^{h} d x-\sum_{T \in \mathcal{T}_{h}} \sum_{j=1}^{J} \omega_{T_{j}} \sum_{i j}\left(a_{i j} \partial_{i} u^{h} \partial_{j} w^{h}\right)\left(x_{T_{j}}\right)\right| \\
& \leq C h^{2 m}\left(\sum_{i j}\left\|a_{i j}\right\|_{W^{2 m, \infty}(\mathcal{D})}\right)\|u\|_{H^{m+1}(\mathcal{D})}\|w\|_{H^{m+1}(\mathcal{D})} .
\end{aligned}
$$

